Article

\title{
Tuning of a Linear-Quadratic Stabilization System for an Anti-Aircraft Missile
}

\author{
Witold Bużantowicz (D)
}

check for

updates

Citation: Bużantowicz, W. Tuning of a Linear-Quadratic Stabilization System for an Anti-Aircraft Missile. Aerospace 2021, 8, 48. https:// doi.org/10.3390/aerospace8020048

Academic Editors: Mark Lowenberg and Earl H. Dowell

Received: 30 September 2020

Accepted: 9 February 2021

Published: 12 February 2021

Publisher's Note: MDPI stays neutral with regard to jurisdictional claims in published maps and institutional affiliations.

Copyright: (C) 2021 by the author. Licensee MDPI, Basel, Switzerland. This article is an open access article distributed under the terms and conditions of the Creative Commons Attribution (CC BY) license (https:/ / creativecommons.org/licenses/by/ $4.0 /)$.
Faculty of Mechatronics, Armament and Aerospace, Military University of Technology, Gen. S. Kaliskiego 2 Street, 00-908 Warsaw, Poland; witold.buzantowicz@wat.edu.pl

\begin{abstract}
A description is given of an application of a linear-quadratic regulator (LQR) for stabilizing the characteristics of an anti-aircraft missile, and an analytical method of selecting the weighting elements of the gain matrix in feedback loop is proposed. A novel method of LQR tuning via a single parameter $\varsigma$ was proposed and tested. The article supplements and develops the topics addressed in the author's previous work. Its added value includes the observation that the solutions obtained are symmetric pairs, and that the tuning parameter $\varsigma$ proposed for the designed linear-quadratic regulator enables the selection of suitable parameters for the airframe stabilizing loop for the majority of the analytical solutions of the considered Riccati equation.
\end{abstract}

Keywords: linear-quadratic regulator; tuning parameter; autopilot; anti-aircraft missile

\section{Introduction}

In anti-aircraft missile homing systems equipped with both mechanically and electronically controlled seekers, one observes a phenomenon of the impact of the airframe oscillations on the operation of the tracking systems. Due to the imperfect disturbance rejection performance, missile motion may severely degrade the tracking accuracy [1-5]. This situation is particularly disadvantageous during the terminal guidance phase, as it requires a very rapid reaction on the part of the missile, involving significant rotations of its airframe. In extremely unfavorable tactical conditions-despite the use of an adequate seeker stabilization loop-the target tracking process may break. It therefore becomes necessary to identify solutions enabling stabilization of the operating conditions of the on-board seeker [6-8]. They are often designed using adaptive linear control, which has certain benefits.

Adaptive linear control is an important component of autonomous vehicle control systems, in which case linear approximations are useful for analysis and design [9]. Linear theory is successfully used for the analysis and synthesis of control systems even though most real systems are nonlinear. The field of linear systems has been declared many times to be exploited and obsolete from a research point of view, but interest has repeatedly been renewed due to new viewpoints and the introduction of new theories [9,10]. Recently, an interest in linear adaptive control in missile and space technology can be observed, where adaptive-tuned PID [11-14], FPID [11,15-17] and linear-quadratic regulator (LQR) [18-25] controllers are widely proposed.

Recent research has focused on satisfying performance and stability robustness requirements by designing missile autopilots using optimal control theory [18,23]. These requirements for current and future missiles are crucial because of the necessity to use multivariable digital flight control systems [26], which in turn are dictated by the conditions of the modern air battlefield. In this context, LQR state feedback designs are generally perceived as providing good performance characteristics and stability margins, with the availability of the states required for implementation $[18,22]$. Thus, in the literature, linearquadratic regulators are proposed for both integrated missile guidance systems and the stabilization of their individual components. For example, in [19] the use of an LQR was 
considered wherein entries of the weighting matrix were chosen as an inverse function of the range-to-go, on the assumption that it is possible to construct a feedback linearized dynamic system from a simulation model of the missile at every value of the state automatically. In [20], a time-varying LQR design method was proposed to deal with the various disturbances in the hypersonic vehicle reentry profile, by calculating the weighting matrices constructed based on the Bryson principle using time-varying parameters. As opposed to classical approaches, the actual states of the flight system were employed to calculate the parameters in weighting matrices. The LQR method is also applied in [21] as a solution for the anti-tank missile guiding process and determines the commands controlling the fin deflections and the thrust vector via continuously calculating Jacobians. In turn, in [18], the LQR formulation is the same, but it was extended to describe the full-state feedback robust integral servo controller equations. The article [24] illustrates that in order to stabilize a highly unstable missile airframe and achieve the required performance, a hybrid of two control schemes may be used. In this case, a state feedback linear quadratic regulator was proposed to stabilize the plant and a forward path $H_{\infty}$ optimal controller was used to achieve the required performance and robustness. Several papers describe the use of an LQR as a part of the missile stabilization system; e.g., [25] discusses a cascaded LQR controller for effective roll stabilization of the missile autopilot in a realistic model; robust missile roll autopilot design with an LQR application for solving the flexible airframe dynamics is also discussed in [23]; and in [7] LQR was chosen as an equivalent part of the sliding mode control (SMC) airframe stabilization system. These selected examples show the multitude of possible LQR applications in aerospace and missile technology.

In the case of a linear time-invariant (LTI) system, the gain coefficients of a linearquadratic controller used in a feedback loop can usually be determined without difficulty. The problem becomes more complicated when the system analyzed contains nonstationary and nonlinear features. This situation occurs, among others, when guiding the missile against an aerial target. The lack of stationarity of the airframe dynamics determines the properties of the whole guidance loop, and can lead to a loss of stability of the entire system. In the case under consideration, when solving the Riccati equation to find the gain coefficients for a missile autopilot feedback loop, numerical computation methods can be applied to a set of static values describing the state of the "frozen" system (i.e., at the moment-for a short finite-time horizon). The fundamental difficulty with this approach is the necessity for the computations to be carried out in real time $[19,27]$. There are also certain technical hitches related to the software implementation, e.g., the weak conditioning of the matrices $[7,8]$. It is therefore valuable to find an analytical solution of the controller, which ensures adaptability according to the state of the system.

In this paper, an adaptive linear-quadratic stabilization system is considered for which a novel method of tuning via a single tuning parameter was proposed and tested. Reducing the stabilization system settings to the case of a single parameter seems attractive from a practical point of view. The main effort is focused on analyzing obtained solutions in detail and describing a procedure for selecting appropriate values of the LQR tuning parameter for the proposed airframe stabilization system. In the author's previous work, an initial concept of achieving an analytical solution of a time-varying linear-quadratic stabilization system was proposed and tested. A basic formula of feedback loop gain matrix equations (without a tuning parameter) was introduced and used as a part of the SMC stabilization system in [7].

It has been shown that the proposed solution provides a favorable effect on the operating conditions of the seeker and a reduction in energy losses resulting from oscillations of the airframe. In turn, [8] signaled the possibility of introducing a single tuning parameter and improving the performance of the designed airframe stabilization system. The main contributions of this paper include: (a) a comparative analysis of the analytical solutions of a time-varying linear-quadratic airframe stabilization system; (b) a procedure for selecting appropriate tuning parameter values; and (c) an observation that the solutions obtained are symmetric pairs, which on the one hand confirms the correctness of the calculations, 
and on the other introduces the possibility of narrowing the range of the sought settings of the LQR tuning parameter.

The paper is organized as follows. The problem is formulated in Section 2.1. The missile's airframe dynamics model's description is given in Section 2.2. The draft of the stabilization system design is discussed in Section 2.3. The tuning procedure is presented in Section 2.4. Issues related to numerical computation methods are described in Section 2.5. To show the features of the proposed solutions, numerical simulations were performed, and their results are given in Section 3. Finally, Section 4 offers some conclusions.

\section{Methods}

\subsection{Problem Formulation}

When guiding a missile towards an aerial target, it is requested that

$$
\omega \stackrel{!}{=} \dot{\theta}
$$

namely, the angular rate $\omega$ of the airframe should be equal to the angular rate $\dot{\theta}$ of the missile's velocity vector, and not depend on the dynamics of the missile itself [6-8]. This requirement, impossible to fulfill in practice however, leads to the conclusion that the absolute rotation angle of the airframe should be approximately equal to the absolute rotation angle of the missile velocity vector; i.e.,

$$
\int \omega^{2} d t \approx \int \dot{\theta}^{2} d t
$$

Obtaining equality given by Equation (2) depends on the system's ability to reduce the effect of the components related to the dynamics of the missile airframe. The left side of the Equation (2) will be close to the right side when the transitional processes occurring during guidance are shorter and less oscillatory. Minimizing the value of the left side of Equation (2) is the task of the designed adaptive linear-quadratic stabilization system.

In the classical approach, the infinite horizon LQR settings are determined based on linear dynamic equations and a quadratic cost function in the form

$$
J=\frac{1}{2} \int_{0}^{\infty}\left[\mathbf{x}^{\mathrm{T}} \mathbf{Q} \mathbf{x}+\mathbf{u}^{\mathrm{T}} \mathbf{R} \mathbf{u}\right] d t
$$

where $\mathbf{Q} \in \mathbb{R}^{n \times n}$ and $\mathbf{R} \in \mathbb{R}^{k \times k}$ are symmetric, positive (semi-positive) definite matrices of weighting parameters for the state vector $\mathbf{x} \in \mathbb{R}^{n \times 1}$ and the control vector $\mathbf{u} \in \mathbb{R}^{k \times 1}$, respectively. In general, there are no unified methods of selecting the $\mathbf{Q}$ and $\mathbf{R}$ entries; however, several approaches can be found in the literature [21,27-36], usually based on modified (e.g., time-varying) Bryson principle or "trial and error" methods. The main problem connecting all of these is the need to define the values of $\mathbf{Q}$ and $\mathbf{R}$ to find the solution of the feedback gain matrix $\mathbf{K}$.

In this study, determining the entry values of matrix $\mathbf{Q}$ is not necessary. Instead, a novel method is proposed by introducing a single tuning parameter $\zeta$ into equations describing the entries of gain matrix $\mathbf{K}$, allowing the change of LQR performance. A linearquadratic controller is applied to a cruciform, canard-controlled, roll-stabilized missile, treated as a SIMO (single-input, multi-output) system, in which control command $u$ is the input signal and the airframe angular rate $\omega$ and angle of attack $\alpha$ are the output signals. The motion of such a missile can be separated into two perpendicular channels, and the problem can be treated as planar in each of these channels.

\subsection{Missile Airframe Dynamics}

As mentioned above, the study relates to a cruciform, canard-controlled, roll-stabilized missile. The stabilization loop in the roll control plane is assumed to be ideal. Using this assumption, its motion can be separated into two perpendicular channels and analyzed 
independently. The equation of motion relative to an axis normal to the velocity vector $\mathbf{V}$ is obtained by projecting the forces acting on the missile onto that axis. The equation of rotational motion is obtained by balancing the moments of forces acting on the missile. This is a typical approach to missile dynamics model determination and it can be found in the form presented or a similar form in a number of research papers [6,37-46]. Neglecting the gravitational force, for the after-burning phase of flight, the dynamics of the missile in the control plane are described by the equations:

$$
\begin{gathered}
m V \dot{\theta}=\frac{\rho V^{2}}{2} S c_{L}^{\alpha} \alpha \\
I \dot{\omega}=-\frac{\rho V^{2}}{2} S l c_{M}^{\alpha} \alpha-\frac{\rho V}{2} S l^{2} c_{M}^{\omega} \omega+\frac{\rho V^{2}}{2} S l c_{M}^{\delta} \delta \\
m \dot{V}=-\frac{\rho V^{2}}{2} S\left[c_{0}+\mu\left(c_{L}^{\alpha} \alpha\right)^{2}\right] \\
\dot{\delta}=\frac{1}{\tau_{c}}\left(\delta_{c o m}-\delta\right)
\end{gathered}
$$

The symbols in Equations (4)-(7) have the following meanings: $m$ is the missile mass $(\mathrm{kg}) ; I$ is the missile moment of inertia $\left(\mathrm{kg} \cdot \mathrm{m}^{2}\right) ; V$ is the module missile velocity vector $(\mathrm{m} / \mathrm{s}) ; \omega$ is the airframe angular rate $(\mathrm{rad} / \mathrm{s}) ; \alpha$ is the angle of attack ( $\mathrm{rad}) ; \theta$ is the angle of the missile velocity vector in control plane ( $\mathrm{rad}) ; S$ is the reference area $\left(\mathrm{m}^{2}\right) ; l$ is the reference length $(\mathrm{m}) ; \rho$ is the air density $\left(\mathrm{kg} / \mathrm{m}^{3}\right) ; \tau_{c}$ is the canard servo time constant $(\mathrm{s}) ; \delta$ and $\delta_{\text {com }}$ are actual and commanded canard deflections (rad), respectively; $\mu$ is induced drag coefficient (-); and $c_{0}$ is the zero-lift drag coefficient $(-)$. The variables described by the common symbol $c_{(\cdot)}^{(\cdot)}$ represent derivatives of the missile nonlinear aerodynamic coefficients, where subscript $M$ describes moment related components and subscript $L$ is the lift force related component, as follows:

$$
c_{L}^{\alpha}=\frac{\partial c_{L}}{\partial \alpha} \quad c_{M}^{\alpha}=\frac{\partial c_{M}}{\partial \alpha} \quad c_{M}^{\omega}=\frac{\partial c_{M \omega}}{\partial \omega} \quad c_{M}^{\delta}=\frac{\partial c_{M}}{\partial \delta}
$$

The force and moment coefficients describing the airframe aerodynamics have complex forms, whose derivations lie outside the scope of this work and will not be considered here. Polynomial approximations $f_{i}, i \in\{1, \ldots, 4\}$ are taken for Mach's number $M a>1.15$, and the following are assumed to hold

$$
f_{1} \approx \frac{\partial c_{L}}{\partial \alpha} \quad f_{2} \approx \frac{\partial c_{M}}{\partial \alpha} \quad f_{3} \approx \frac{\partial c_{M \omega}}{\partial \omega} \quad f_{4} \approx \frac{\partial c_{M}}{\partial \delta}
$$

Noting that

$$
\alpha \equiv \vartheta-\theta \quad \rightarrow \quad \dot{\alpha} \equiv \dot{\vartheta}-\dot{\theta}=\omega-\dot{\theta}
$$

where $\vartheta$ is the airframe rotation angle in the control plane (rad), the following equivalent for Equations (4) and (5) can be obtained:

$$
\begin{gathered}
\dot{\alpha}=-\frac{\rho V}{2 m} S f_{1} \alpha+\omega \\
\dot{\omega}=-\frac{\rho V^{2}}{2 I} S l f_{2} \alpha-\frac{\rho V}{2 I} S l^{2} f_{3} \omega+\frac{\rho V^{2}}{2 I} S l f_{4} \delta
\end{gathered}
$$

For controller design purposes, and for reasons of simplicity, it is assumed that $u=$ $\delta_{\text {com }}=\delta$; i.e., the inertia of the canards is ignored. Taking this into account, approximate dynamics, which will be used for the design of the linear-quadratic stabilization system, can be described by linearized vector-matrix equations as

$$
\dot{\mathbf{x}}=\mathbf{A x}+\mathbf{B} u \quad \mathbf{y}=\mathbf{C} \mathbf{x}+\mathbf{D} u
$$


where

$$
\mathbf{x}=\left[\begin{array}{c}
\alpha \\
\omega
\end{array}\right] \quad \mathbf{A}=\left[\begin{array}{cc}
-a_{1} & 1 \\
-a_{2} & -a_{3}
\end{array}\right] \quad \mathbf{B}=\left[\begin{array}{l}
0 \\
b
\end{array}\right] \quad \mathbf{C}=\left[\begin{array}{ll}
1 & 0 \\
0 & 1
\end{array}\right] \quad \mathbf{D}=\left[\begin{array}{l}
0 \\
0
\end{array}\right]
$$

The entries of matrices $\mathbf{A}$ and $\mathbf{B}$ are assumed to have the following forms:

$$
a_{1}=\frac{\rho V}{2 m} S f_{1} \quad a_{2}=\frac{\rho V^{2}}{2 I} S l f_{2} \quad a_{3}=\frac{\rho V}{2 I} S l^{2} f_{3} \quad b=\frac{\rho V^{2}}{2 I} S l f_{4}
$$

\subsection{Stabilization System Design}

Using Equation (3), by the variation method of the optimal control theory, the feedback gain matrix $\mathbf{K}$ can be obtained as follows:

$$
\mathbf{K}=\mathbf{R}^{-1} \mathbf{B}^{\mathrm{T}} \mathbf{P}
$$

where $\mathbf{P}$ is the stabilizing solution of the Riccati equation

$$
\mathbf{A}^{\mathrm{T}} \mathbf{P}+\mathbf{P A}-\mathbf{P B R}^{-1} \mathbf{B}^{\mathrm{T}} \mathbf{P}+\mathbf{Q}=\mathbf{0}
$$

Let us take the matrices $\mathbf{Q}$ and $\mathbf{R}$ to be

$$
\mathbf{Q}=\left[\begin{array}{cc}
q_{1} & 0 \\
0 & q_{2}
\end{array}\right] \quad \text { and } \quad \mathbf{R}=1
$$

Scaling $\mathbf{R}$ for single-input systems results only in the same amount of scaling on $\mathbf{Q}$. In the case under consideration, $\mathbf{R}=1$ was therefore chosen. Assuming that

$$
\mathbf{P}=\left[\begin{array}{ll}
p_{11} & p_{12} \\
p_{21} & p_{22}
\end{array}\right]
$$

the expanded form of the Equation (17) can be obtained as

$$
\begin{gathered}
{\left[\begin{array}{cc}
-a_{1} p_{11}-a_{2} p_{21} & -a_{1} p_{12}-a_{2} p_{22} \\
p_{11}-a_{3} p_{21} & p_{12}-a_{3} p_{22}
\end{array}\right]+\left[\begin{array}{cc}
-a_{1} p_{11}-a_{2} p_{12} & p_{11}-a_{3} p_{12} \\
-a_{1} p_{21}-a_{2} p_{22} & p_{21}-a_{3} p_{22}
\end{array}\right]+} \\
-b^{2}\left[\begin{array}{ll}
p_{12} p_{21} & p_{12} p_{22} \\
p_{21} p_{22} & p_{22} p_{22}
\end{array}\right]+\left[\begin{array}{cc}
q_{1} & 0 \\
0 & q_{2}
\end{array}\right]=\left[\begin{array}{ll}
0 & 0 \\
0 & 0
\end{array}\right]
\end{gathered}
$$

In previous work $[7,8]$, a complete solution was given for the controller in question. Here, a draft is presented for the sake of brevity. By summation of the matrices in Equation (20), rearrangement and while noting that $p_{12} \equiv p_{21}$, the system of equations takes the form:

$$
\left\{\begin{array}{l}
-2 a_{1} p_{11}-2 a_{2} p_{21}+q_{1}=b^{2} p_{21}^{2} \\
p_{11}-\left(a_{1}+a_{3}\right) p_{21}-a_{2} p_{22}=b^{2} p_{21} p_{22} \\
2 p_{21}-2 a_{3} p_{22}+q_{2}=b^{2} p_{22}^{2}
\end{array}\right.
$$

By multiplying both sides of the second line in Equation (21) by 2 and summing, we have

$$
\left(1-a_{1}\right) p_{11}+\left(1-a_{1}-a_{2}-a_{3}\right) p_{21}+\left(-a_{2}-a_{3}\right) p_{22}=b^{2}\left(p_{21}+p_{22}\right)^{2}-q_{1}-q_{2}
$$

The values of $q_{1}$ and $q_{2}$ are positive-defined and can be freely chosen by the designer. Let us assume that

$$
\left(p_{21}+p_{22}\right)^{2}-\frac{\varsigma^{2}}{b^{2}}=0 \quad \text { where } \quad \varsigma=q_{1}+q_{2}
$$


There are two possible solutions of Equation (23)

$$
p_{21}=\frac{\varsigma}{b}-p_{22} \quad \text { and } \quad p_{21}=-\frac{\varsigma}{b}-p_{22}
$$

By using Equation (24) to solve the system in Equation (21), with Equation (16), after some transformations, eight pairs of entries for the matrix $\mathbf{K}$ can be found as follows:

$$
\begin{aligned}
\mathbf{K}_{1,2}= & {\left[\begin{array}{c}
-\frac{1}{b\left(1-2 a_{1}\right)}\left[a_{1}\left(a_{1}-a_{2}+a_{3}+b\right)+a_{2} \pm \sqrt{\Psi_{1}}\right] \\
\varsigma+\frac{1}{b\left(1-2 a_{1}\right)}\left[a_{1}\left(a_{1}-a_{2}+a_{3}+b\right)+a_{2} \pm \sqrt{\Psi_{1}}\right]
\end{array}\right]^{\mathrm{T}} } \\
\mathbf{K}_{3,4}= & {\left[\begin{array}{c}
-\frac{1}{b\left(1-2 a_{1}\right)}\left[a_{1}\left(a_{1}-a_{2}+a_{3}-b\right)+a_{2} \pm \sqrt{\Psi_{2}}\right] \\
-\varsigma+\frac{1}{b\left(1-2 a_{1}\right)}\left[a_{1}\left(a_{1}-a_{2}+a_{3}-b\right)+a_{2} \pm \sqrt{\Psi_{2}}\right.
\end{array}\right]^{\mathrm{T}} } \\
\mathbf{K}_{5,6}= & {\left[\begin{array}{c}
\varsigma+\frac{1}{b}\left[\left(a_{3}+1\right) \mp \sqrt{a_{3}^{2}+2 a_{3}+\frac{1}{2} b^{2}+2 b+1}\right] \\
-\frac{1}{b}\left[\left(a_{3}+1\right) \pm \sqrt{a_{3}^{2}+2 a_{3}+\frac{1}{2} b^{2}+2 b+1}\right]
\end{array}\right] } \\
\mathbf{K}_{7,8}= & {\left[\begin{array}{c}
\mathrm{T} \\
-\varsigma+\frac{1}{b}\left[\left(a_{3}+1\right) \mp \sqrt{a_{3}^{2}+2 a_{3}+\frac{1}{2} b^{2}-2 b+1}\right. \\
-\frac{1}{b}\left[\left(a_{3}+1\right) \pm \sqrt{a_{3}^{2}+2 a_{3}+\frac{1}{2} b^{2}-2 b+1}\right]
\end{array}\right]^{\mathrm{T}} }
\end{aligned}
$$

where $\varsigma$ is treated as a tuning parameter of the proposed controller, and $\Psi_{1}$ and $\Psi_{2}$ are functions given as

$$
\begin{gathered}
\Psi_{1}=\left(a_{1} b+a_{2}\right)^{2}+\left[a_{1}\left(a_{1}+a_{3}\right)-a_{1} a_{2}\right]^{2}+2\left(a_{1}-a_{2}+a_{3}\right)\left(a_{1}^{2} b+a_{1} a_{2}\right)+ \\
+\left(\frac{1}{2}-a_{1}\right)\left(b^{2}-4 a_{1} a_{2} b\right) \\
\Psi_{2}=\left(a_{1} b-a_{2}\right)^{2}+\left[a_{1}\left(a_{1}+a_{3}\right)-a_{1} a_{2}\right]^{2}-2\left(a_{1}-a_{2}+a_{3}\right)\left(a_{1}^{2} b-a_{1} a_{2}\right)+ \\
+\left(\frac{1}{2}-a_{1}\right)\left(b^{2}+4 a_{1} a_{2} b\right)
\end{gathered}
$$

Based on Equation (16) and Equations (25)-(30), the control law can be defined as

$$
u_{L Q R}=\left(N_{u}+\mathbf{K N}_{x}\right) u-\mathbf{K x}
$$

where $\mathbf{K}$ is one of the feedback gain matrices given by Equations (25)-(28). The following expressions are used as the feed-forwarding scaling factors of the input signal (cf. Appendix A):

$$
\left[\begin{array}{l}
\mathbf{N}_{x} \\
N_{u}
\end{array}\right]=\mathbf{\Omega}^{-1}\left[\begin{array}{l}
0 \\
1
\end{array}\right] \quad \text { where } \quad \boldsymbol{\Omega}=\left[\begin{array}{ccc}
-a_{1} & 1 & 0 \\
-a_{2} & -a_{3} & b \\
0 & 1 & 0
\end{array}\right]
$$

with $\operatorname{det}(\boldsymbol{\Omega})=a_{1} b$. Due to the application specifics, the condition $a_{1} b>0$ is always met. 


\subsection{LQR Tuning Procedure}

The choice of the tuning parameter $\varsigma$ value for the particular feedback loop gain matrix $\mathbf{K}$ is determined on the basis of Equation (2) and three additional quantity indices, defined as

$$
J_{\mathrm{A}}=\sum_{n=1}^{N} \mathrm{~A}(n) \quad J_{\Lambda}=\sum_{n=0}^{N} \Lambda(n) \quad J_{\mathrm{X}}=\sum_{n=0}^{N} \mathrm{X}(n)
$$

in which $\mathrm{A}, \Lambda$ and $\mathrm{X}$ are given as

$$
\begin{gathered}
\mathrm{A}(n)=\left\{\begin{array}{lll}
0 & \Leftrightarrow & a(n) \geq a(n-1) \\
1 & \Leftrightarrow & a(n)<a(n-1)
\end{array}\right. \\
\Lambda(n)=\left\{\begin{array}{lll}
0 & \Leftrightarrow & \alpha(n) \leq \alpha(N) \\
1 & \Leftrightarrow & \alpha(n)>\alpha(N)
\end{array}\right. \\
\mathrm{X}(n)=\left\{\begin{array}{l}
1 \\
0
\end{array} \quad \alpha(n)<0.95 \alpha(N) \wedge n>n_{\text {bound }} \wedge\right.
\end{gathered}
$$

where $a$ is the normal acceleration of the airframe $\left(\mathrm{m} / \mathrm{s}^{2}\right), \alpha$ is the angle of attack (rad) and $(N+1)$ is the number of signal samples considered.

The quantity index $J_{\mathrm{A}}$ enables the detection of oscillations of the airframe in response to a step canard-fin deflection: in the case where the instantaneous value of the normal acceleration of the airframe is smaller than the value of that acceleration at the previous instant, the value of $J_{\mathrm{A}}$ is increased by 1 .

The index $J_{\Lambda}$ is used to detect over-regulation in the stabilization system. Overregulation is examined based on the angle of attack $\alpha$ (in view of the dynamic properties of the system, it is inappropriate to detect over-regulation using the airframe's angular rate $\omega$ ). The reference value is taken to be the angle of attack at instant $N$, for which it is known a priori that the steady state of the system was achieved.

The index $J_{X}$, in turn, is useful in determining the speed of the airframe's response to a step canard-fin deflection. The value of the index is increased by 1 for every instant $n$ greater than $n_{\text {bound }}$ for which the airframe's angle of attack $\alpha$ does not attain $95 \%$ of the value of the angle of attack in the steady state $\alpha(N)$. In the numerical simulations, $n_{\text {bound }}$ was set equal to 1500 , which corresponds to a time of $0.15 \mathrm{~s}$ for a sampling frequency of $f_{s}=10 \mathrm{kHz}$.

The tuning parameter $\varsigma$ waa s selected according to the following rules. For chosen value of $\varsigma$ : (a) the absolute airframe rotation angle given by Equation (2) takes the minimum value; (b) the quality indices $J_{\mathrm{A}}$ and $J_{\Lambda}$ take the minimum values in the considered range. The quality index $J_{X}$ plays an auxiliary role and establishes that the response of the airframe in the considered case may be slower than the required one. In the case of missiles number 6 and number 7 , the values of $\varsigma$ were chosen arbitrarily, because the obtained values of the quality indices did not make it possible to clearly indicate the solutions, and the responses of the tested airframes were mostly unstable. To make a decision in this case, the values of the absolute rotation angle obtained for the individual values of the tuning parameter $\varsigma$ were used.

With respect to the tuning procedure, an iterative method was investigated for the response of the missile airframe to stepped canard-fin deflections. Calculations were performed for a set of coefficients defined in Equation (15) with constant values, describing the system state in a short-time horizon, for the instant of commencement of the terminal phase of missile guidance to an aerial target.

As it can be deduced from [7], setting an initial value of $\zeta=1$ gives a good start point for the beginning of the LQR tuning procedure. Figures 1-8 show the results of numerical computations for the different forms of the gain matrix $\mathbf{K}$ implemented in the stabilizing loop, and the values taken for the airframe coefficients: $a_{1}=5 \mathrm{~s}^{-1}, a_{2}=2350 \mathrm{~s}^{-2}$, $a_{3}=10 \mathrm{~s}^{-1}, b=420 \mathrm{~s}^{-2}$. 
In Figures 1-8, the results obtained for the quantity indices defined by Equation (33) are shown only in the range $|\varsigma|<10$, due to the fact that the numerical analysis did not reveal any significant changes in the functioning of the analyzed stabilization systems outside that range (relative to the results attained for the boundary values of the assumed range of variation of the parameter $\varsigma$ ).

The numerical simulations revealed symmetries between the forms of the quantity indices determined for particular gain matrices (compare Figures 1a and 4a, Figures 2a and 3a, Figures $5 a$ and $8 a$, Figures $6 a$ and $7 a$ ). It may thus be assumed that the matrices given by Equations (25)-(28) form symmetric pairs of solutions, namely: $\mathbf{K}_{1}-\mathbf{K}_{4}$ (with the axis of symmetry passing through the point $\left.\zeta_{\text {sym }}=1.25\right), \mathbf{K}_{2}-\mathbf{K}_{3}$ (with axis of symmetry $\varsigma_{\text {sym }}=-0.045$ ), $\mathbf{K}_{5}-\mathbf{K}_{8}$ (with axis of symmetry $\varsigma_{\text {sym }}=0.79$ ) and $\mathbf{K}_{6}-\mathbf{K}_{7}$ (with axis of symmetry $\varsigma_{\text {sym }}=-0.73$ ). This is illustrated in detail in Figures 9-12.

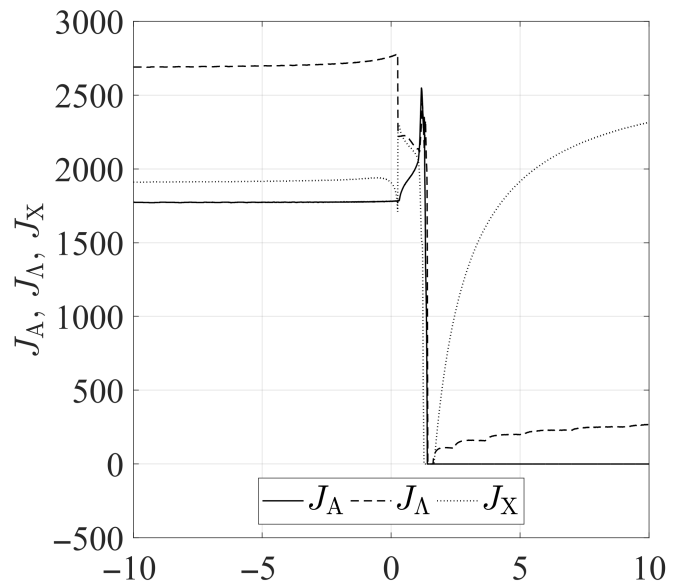

a)

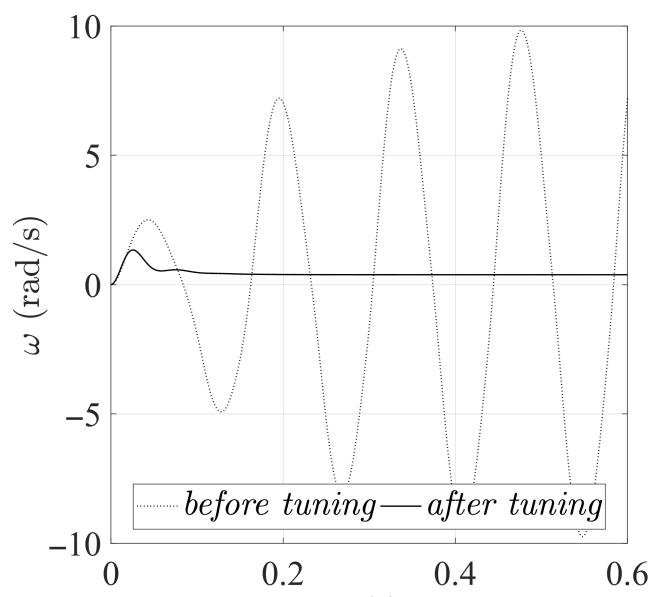

b) $t(\mathrm{~s})$

Figure 1. Tuning procedure results for feedback loop gain matrix $\mathbf{K}_{1}$ : (a) Quantity indices for different $\varsigma$ values. (b) Step responses of the airframe before and after the tuning procedure.

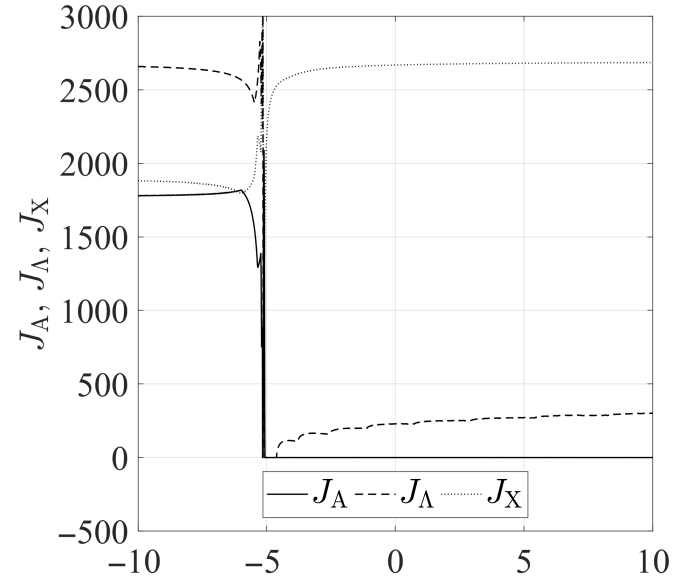

a)

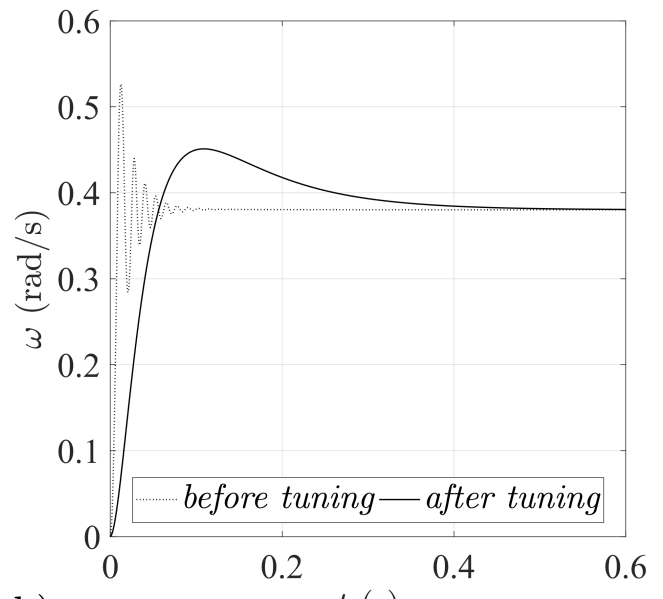

b)

$$
t(\mathrm{~s})
$$

Figure 2. Tuning procedure results for feedback loop gain matrix $\mathbf{K}_{2}$ : (a) Quantity indices for different $\varsigma$ values. (b) Step responses of the airframe before and after the tuning procedure. 


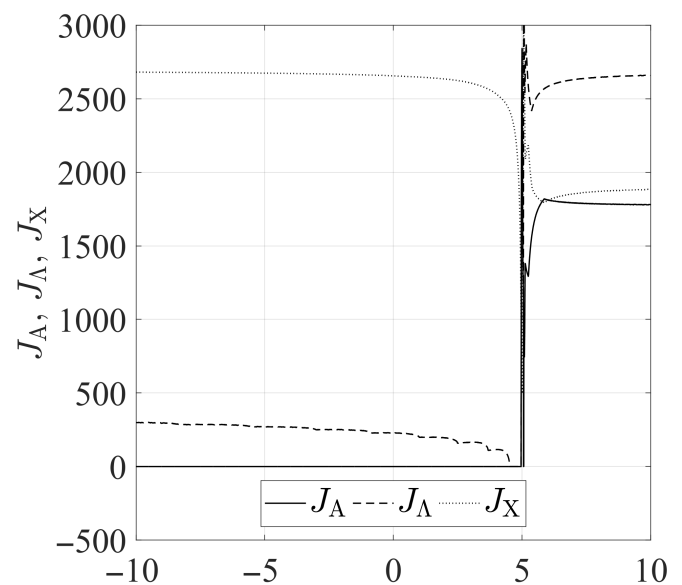

a)

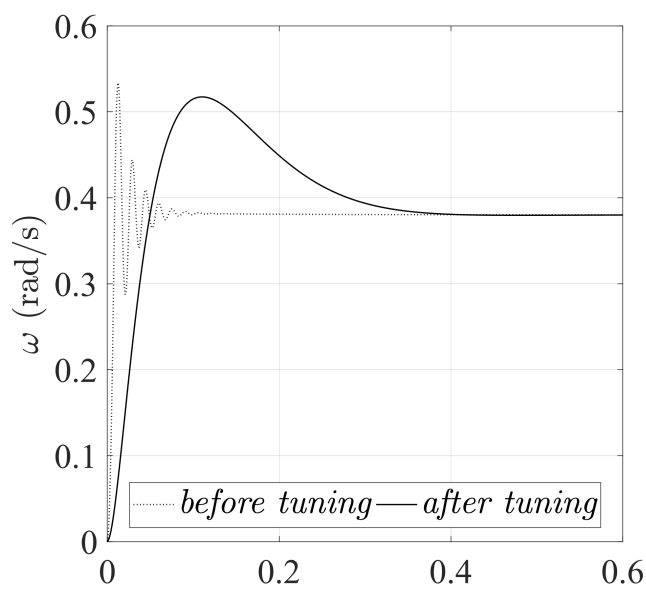

b)

$t(\mathrm{~s})$

Figure 3. Tuning procedure results for feedback loop gain matrix $\mathbf{K}_{3}$ : (a) Quantity indices for different $\varsigma$ values. (b) Step responses of the airframe before and after the tuning procedure.

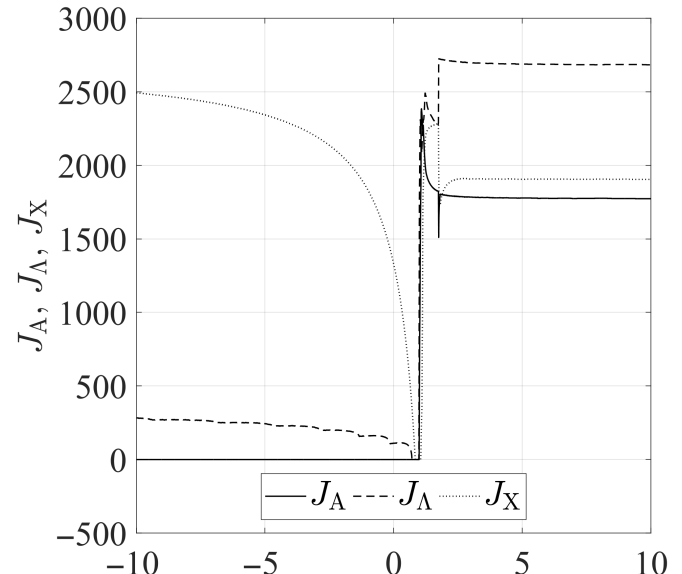

a)

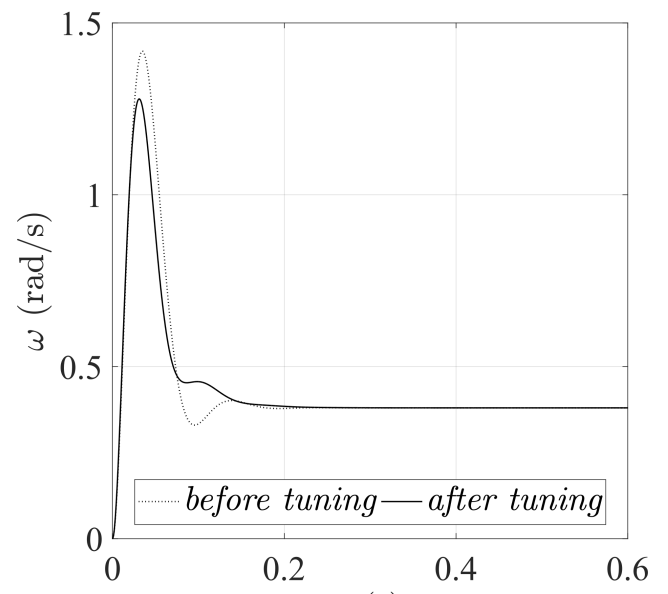

b)

$t(\mathrm{~s})$

Figure 4. Tuning procedure results for feedback loop gain matrix $\mathbf{K}_{4}$ : (a) Quantity indices for different $\varsigma$ values. (b) Step responses of the airframe before and after the tuning procedure.

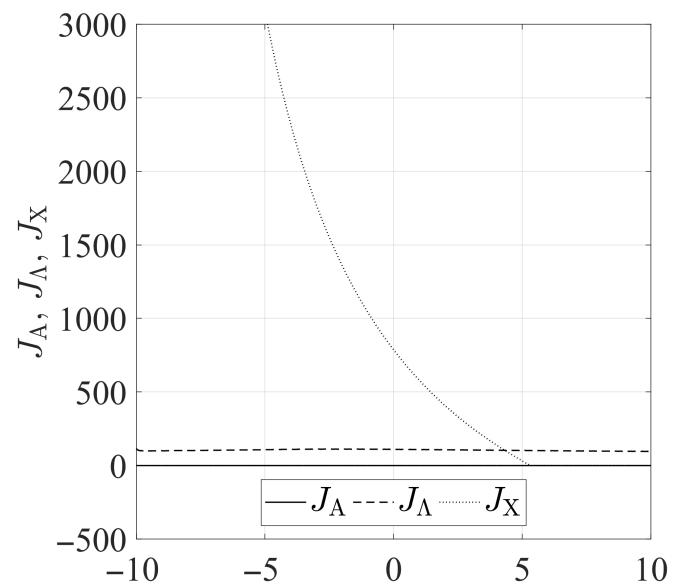

a)

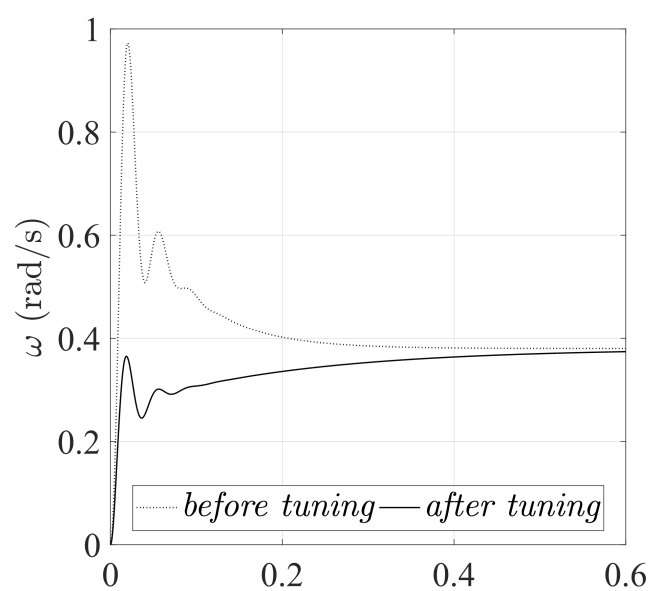

b)

Figure 5. Tuning procedure results for feedback loop gain matrix $\mathbf{K}_{5}$ : (a) Quantity indices for different $\varsigma$ values. (b) Step responses of the airframe before and after the tuning procedure. 


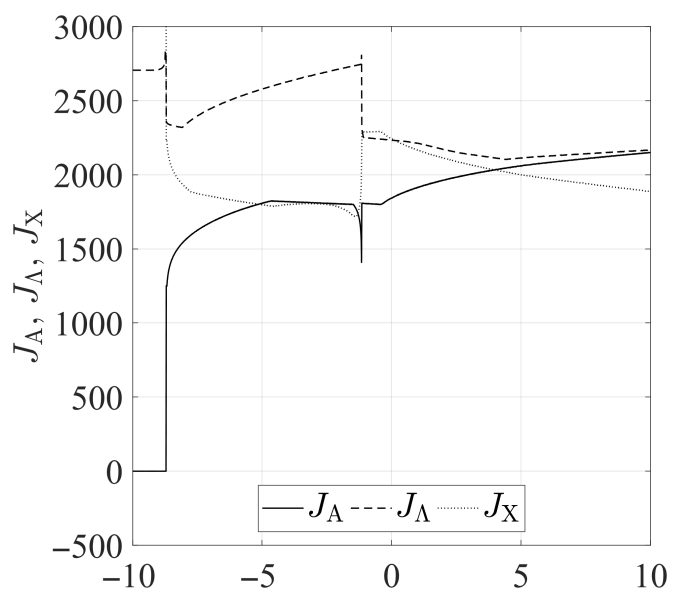

a)

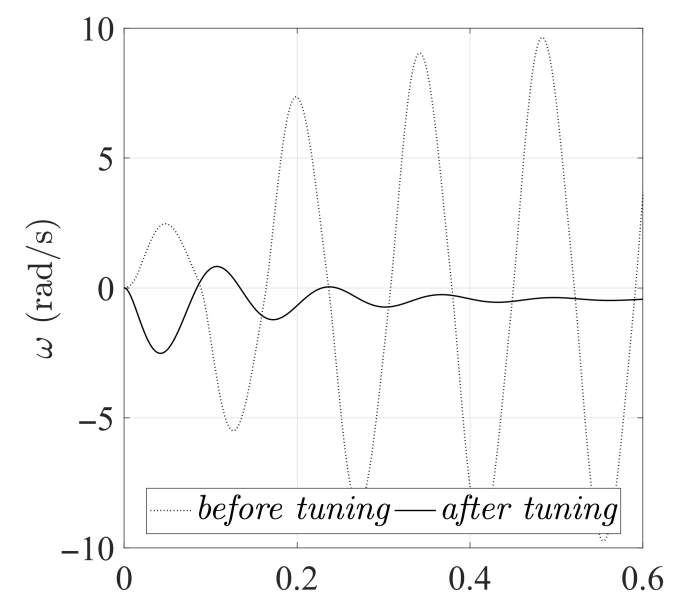

b)

$t(\mathrm{~s})$

Figure 6. Tuning procedure results for feedback loop gain matrix $\mathbf{K}_{6}$ : (a) Quantity indices for different $\varsigma$ values. (b) Step responses of the airframe before and after the tuning procedure.

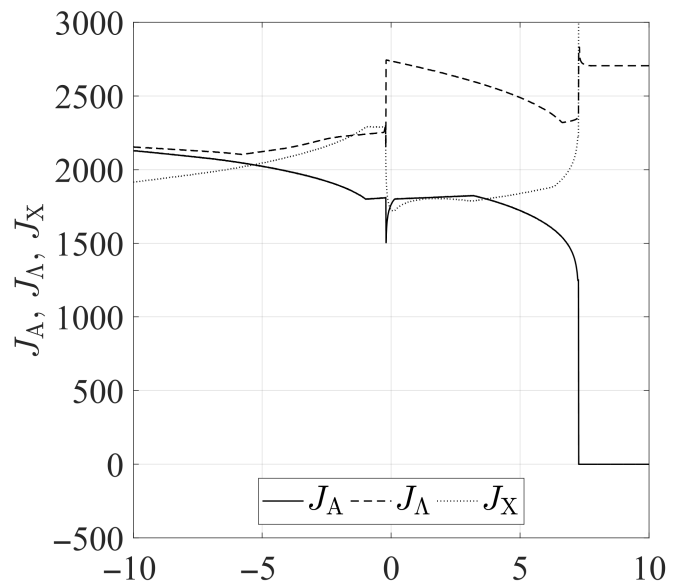

a)

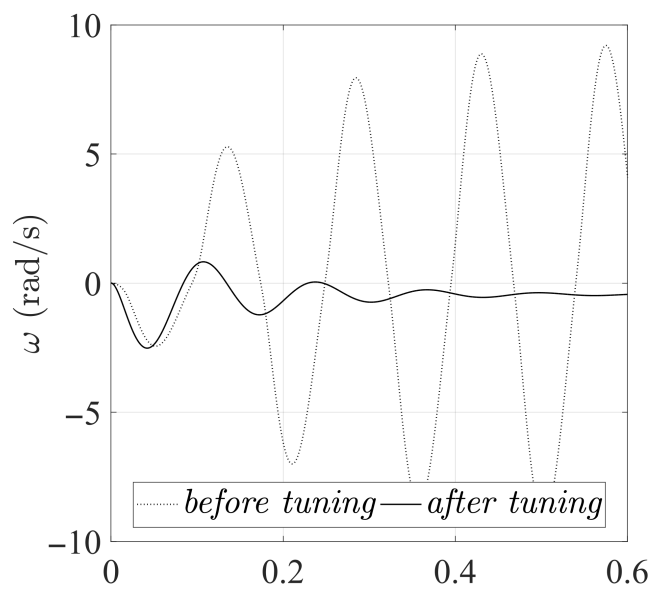

b)

Figure 7. Tuning procedure results for feedback loop gain matrix $\mathbf{K}_{7}$ : (a) Quantity indices for different $\varsigma$ values. (b) Step responses of the airframe before and after the tuning procedure.

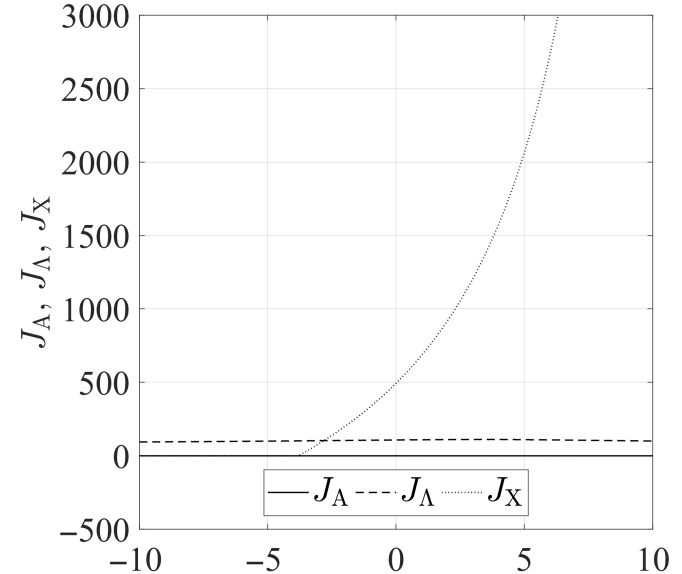

a)

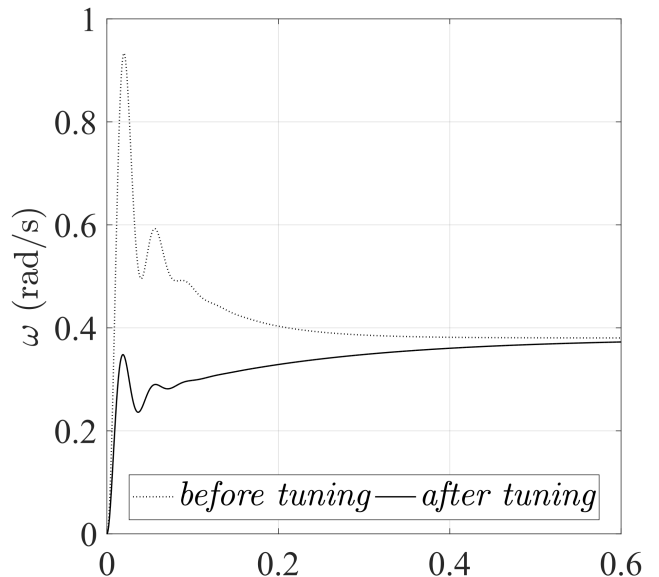

b)

$t(\mathrm{~s})$

Figure 8. Tuning procedure results for feedback loop gain matrix $\mathbf{K}_{8}$ : (a) Quantity indices for different $\varsigma$ values. (b) Step responses of the airframe before and after the tuning procedure. 
This fact suggests that the sought values of the tuning parameter $\varsigma$ should be distributed symmetrically with respect to those axes, i.e., $\zeta_{s y m} \pm \Delta \zeta$. Indeed, the tuning values determined numerically based on the indices (33) for particular forms of the matrices given by Equations (25)-(28) satisfy that relationship (cf. Table 1).

Table 1. Symmetry between gain matrices and tuning parameter values.

\begin{tabular}{|c|c|c|c|c|c|c|}
\hline Gain & Tuning Parameter & \multicolumn{3}{|c|}{ Axis of Symmetry } & \multirow{2}{*}{$\begin{array}{c}\text { Tuning Parameter } \\
\varsigma=\varsigma_{\text {sym }}+\Delta \varsigma\end{array}$} & \multirow{2}{*}{$\begin{array}{c}\text { Gain } \\
\text { Matrix }\end{array}$} \\
\hline Matrix & $\varsigma=\varsigma_{\text {sym }}-\Delta \varsigma$ & $-\Delta s$ & $\varsigma_{\text {sym }}$ & $+\Delta s$ & & \\
\hline $\mathbf{K}_{2}$ & -5.060 & -5.015 & -0.045 & 5.015 & 4.970 & $\mathbf{K}_{3}$ \\
\hline $\mathbf{K}_{4}$ & 0.940 & -0.310 & 1.250 & 0.310 & 1.560 & $\mathbf{K}_{1}$ \\
\hline $\mathbf{K}_{6}$ & -9.190 & -8.455 & -0.735 & 8.455 & 7.720 & $\mathbf{K}_{7}$ \\
\hline $\mathbf{K}_{5}$ & -6.210 & -7.000 & 0.790 & 7.000 & 7.790 & $\mathbf{K}_{8}$ \\
\hline
\end{tabular}
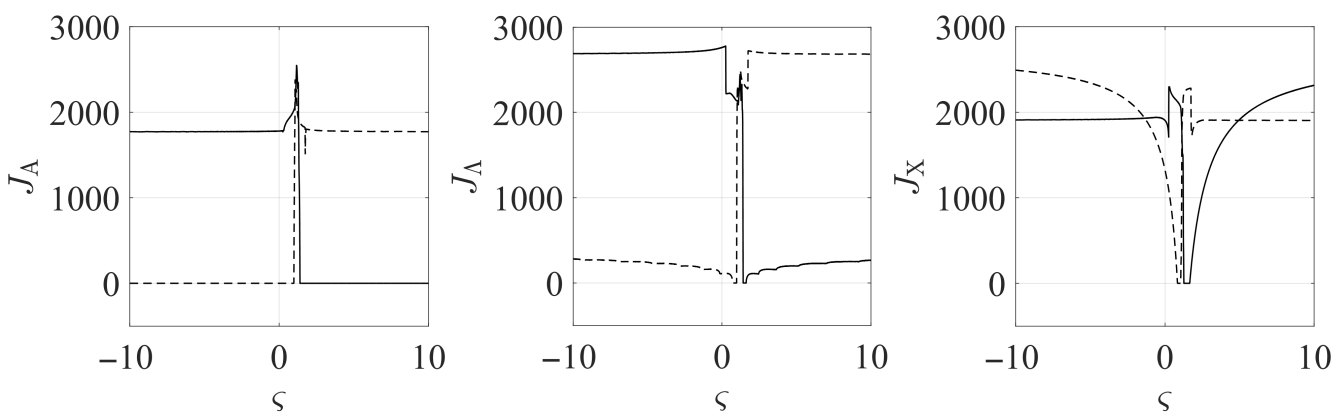

$$
-\mathbf{K}_{1}---\mathbf{K}_{4}
$$

Figure 9. Symmetry between quantity indices of matrices $\mathbf{K}_{1}$ and $\mathbf{K}_{4}$.
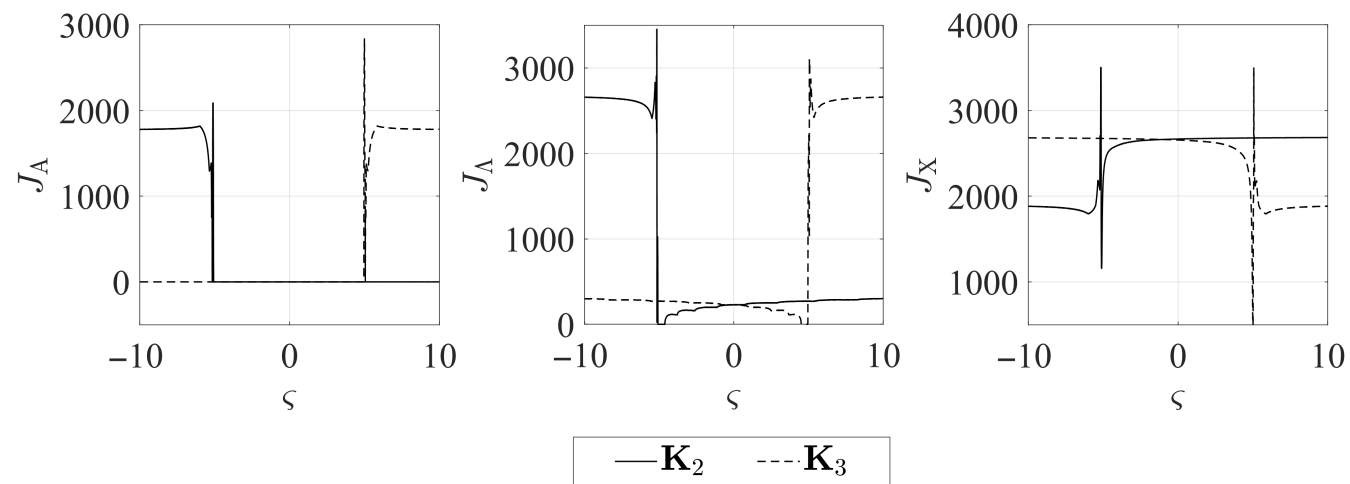

Figure 10. Symmetry between quantity indices of matrices $\mathbf{K}_{2}$ and $\mathbf{K}_{3}$.
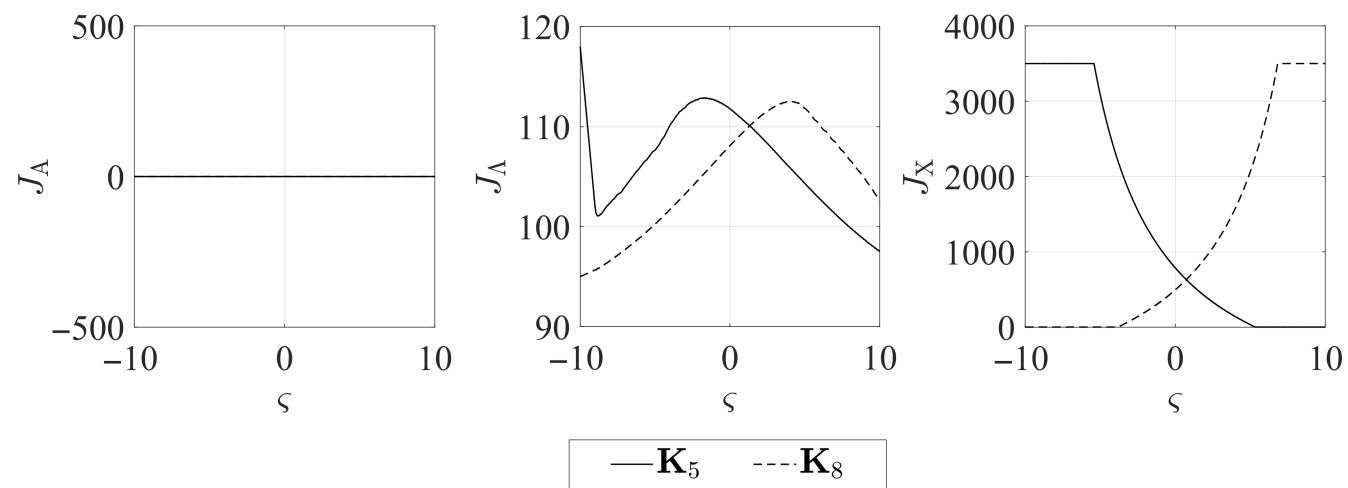

Figure 11. Symmetry between quantity indices of matrices $\mathbf{K}_{5}$ and $\mathbf{K}_{8}$. 

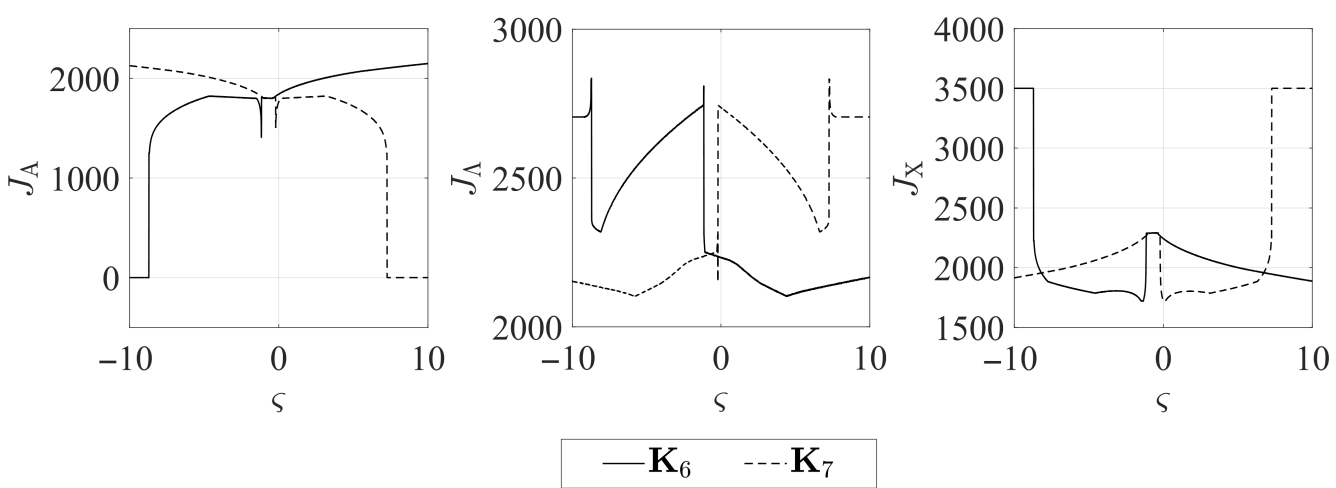

Figure 12. Symmetry between quantity indices of matrices $\mathbf{K}_{6}$ and $\mathbf{K}_{7}$.

Figure 13 shows the graphs of angular rates $\omega$ and angles of attack $\alpha$ obtained as a result of the response of missile airframes with stabilization systems using the matrices $\mathbf{K}_{1}-\mathbf{K}_{8}$ to an applied test signal. A symmetric rectangular signal with values $\pm 0.3 \mathrm{rad}$ and a $50 \%$ duty cycle was input to the system, simulating stepped, alternating canardfin deflections.
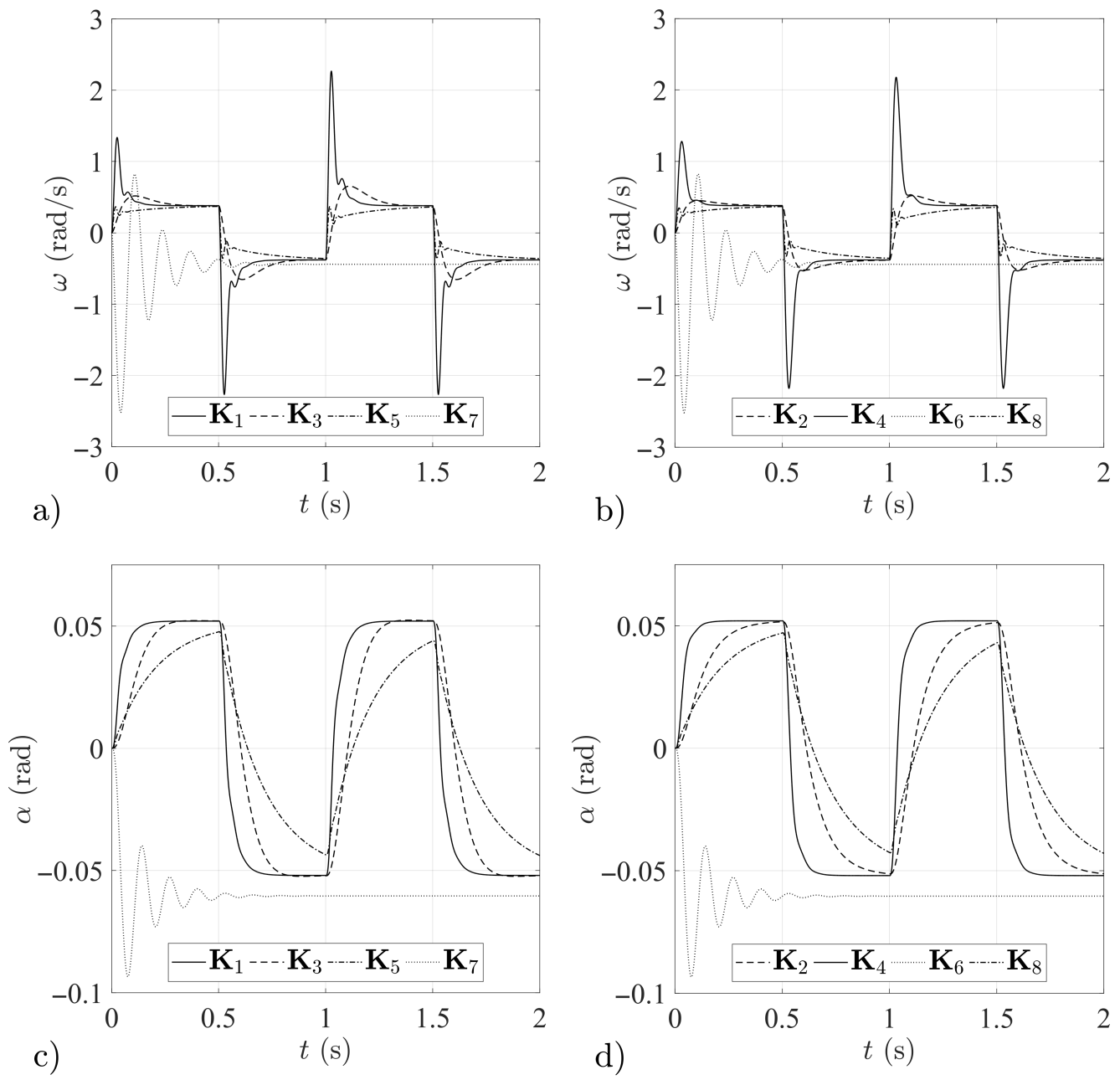

Figure 13. Missile airframe: (a,b) angular rate, $(\mathbf{c}, \mathbf{d})$ angle of attack histories for different feedback loop gain matrices.

The following conclusions can be drawn from the results obtained. 
Stabilization system solutions using the gain matrices $\mathbf{K}_{1}-\mathbf{K}_{4}$ ensure a correct reaction to the applied input signal. In the case of stabilization systems configured based on the matrices $\mathbf{K}_{2}$ and $\mathbf{K}_{3}$, smooth graphs are obtained with the required response dynamics, although it should be noted that the system with the matrix $\mathbf{K}_{2}$ exhibits slightly higher inertia, which may lead to poorer results for missile guidance to an aerial target of high maneuverability. The graphs of airframe angular rates in the case of stabilization systems with the matrices $\mathbf{K}_{1}$ and $\mathbf{K}_{4}$ suggest that missiles with these systems will respond very rapidly to all changes in the input signal, but this may lead to oscillations if changes in the signal are of too high a frequency. In turn, stabilization systems based on the matrices $\mathbf{K}_{5}$ and $\mathbf{K}_{8}$, in spite of the absence of oscillations in the recorded responses, exhibit high inertia, which substantially restricts their possible use in practical solutions.

The simulation results obtained for the systems with matrices $\mathbf{K}_{6}$ and $\mathbf{K}_{\mathbf{7}}$ indicate that these are unsuitable for use in the problem under consideration.

\subsection{Numerical Simulation Issues}

The performance of the proposed gain matrices is evaluated through numerical simulations. The fourth-order Runge-Kutta numerical integration method was used for the derivation of approximating differential equations for the elements of the system. The time of the simulation was $t_{f}=2 \mathrm{~s}$.

The numerical examples presented in Section 3 relate to the missile model with parameters similar to those of modern short-range surface-to-air missiles. The missile's initial mass is $250 \mathrm{~kg}$, and the fuel load was taken as $60 \%$ of the total mass. The rocket engine allows the missile to achieve a maximum speed of $1030 \mathrm{~m} / \mathrm{s}$ with a burning-phase time of 3 seconds. It is assumed that the terminal missile-engagement phase is considered, i.e., the missile has no thrust during the tests. Missile velocity change is described according to the Equation (6). The following additional parameters were assumed in the simulations: $I=35 \mathrm{~kg} \cdot \mathrm{m}^{2}, S=0.67 \mathrm{~m}^{2}$, and $l=1.36 \mathrm{~m}$. Partial derivatives of aerodynamic force and moment coefficients with respect to the variables $\alpha, \delta$ and $\omega$, and a zero-lift drag coefficient were determined on the basis of the classical methodology outlined among others in [47-49] and approximated by polynomial functions, as shown by Equation (9). The canard deflection angle is bounded to $|\delta|<0.35$ rad and the canard servo time constant is taken as $\tau_{c}=0.01 \mathrm{~s}$, cf. Equation (7). The atmosphere has been modelled in compliance with International Standard Atmosphere.

Data supply for the coordinate determination system is provided by the simplified missile seeker model given by Equation (37). The signal returned by the seeker contains information about the angular rate of the line of sight (LOS) distorted by the inertia of the seeker system, by the signal proportional to the angular rate of the airframe, and by generally understood measurement errors:

$$
\dot{\lambda}_{m}=\frac{1}{\tau_{s}}\left(\lambda+\Delta \lambda+\zeta \omega-\lambda_{m}\right)
$$

where $\lambda$ and $\lambda_{m}$ are the actual and measured LOS angles (rad); $\zeta=0.1 \mathrm{~ms}$ is the sampling period; $\tau_{s}=5 \mathrm{~ms}$ is the time constant of the seeker drives; $\Delta \lambda$ denotes the fluctuating interference caused by the noise from on-board devices, mechanical vibrations, and the environment, and is modelled as white noise with Gaussian distribution.

A classical form of proportional navigation law is applied to guide the missiles to the aerial target [37-39]. The target is assumed as a mass point moving at a constant velocity. Its first-order lateral manoeuvre dynamics is expressed in the control plane by

$$
\dot{a}_{T}=\frac{1}{\tau_{T}}\left(u_{T}-a_{T}\right)
$$

where $\tau_{T}$ is the time constant of the target dynamics (s), $u_{T}$ is the manoeuvre command $\left(\mathrm{m} / \mathrm{s}^{2}\right)$, and $a_{T}$ is the lateral acceleration $\left(\mathrm{m} / \mathrm{s}^{2}\right)$ of the aerial target (cf. Appendix B for details). 


\section{Results and Discussion}

The proposed gain matrix formulas were examined for the terminal guidance phase. A comparison of the guidance processes was made for the eight missile models using the feedback loop gain matrices given by Equations (25)-(28) with the tuning parameter values presented in Section 2.4 (cf. Table 1). First, a basic examination of the proposed structures was performed using the sample run test. Next, a Monte Carlo simulation study was carried out to evaluate and compare the performance of the solutions.

\subsection{Sample Run Test}

The missiles are aimed at an aerial target maneuvering with sinus-wave lateral acceleration $a_{T}= \pm 10 \mathrm{~g}$ in the yaw plane. Target dynamics is described by time constant $\tau_{T}=1 \mathrm{~s}$. At time $t=0$ the target is located at course angle $\gamma_{T}=3 \pi / 4 \mathrm{rad}$ and is moving at $V_{T}=220 \mathrm{~m} / \mathrm{s}$ at a height of $4000 \mathrm{~m}$ and a distance of $1800 \mathrm{~m}$ from the initial position of the missiles. At $t=0$ the missiles have a height of $4000 \mathrm{~m}$, the yaw angle of the airframe is equal to the yaw angle of the velocity vector $\vartheta=\theta=0 \mathrm{rad}$, and the initial velocity is $V=950 \mathrm{~m} / \mathrm{s}$. According to Equation (2), absolute rotation angle $\Theta$ is defined by

$$
\Theta=\sum_{n=0}^{N}\left[\frac{1}{f_{s}}|\omega(n)|\right] \quad \text { where } \quad N=f_{s} t_{g}
$$

in which $n$ is the sample index, $t_{g}$ is the guidance time, and $f_{s}=10 \mathrm{kHz}$ is the sampling frequency. Guidance time $t_{g}$ is understood as the time elapsed between $t=0$ and the instant at which the closing velocity changes the sign. The miss distance $d$ is defined as

$$
d=\sqrt{\left(x_{T}-x\right)^{2}+\left(y_{T}-y\right)^{2}+\left(z_{T}-z\right)^{2}}
$$

where $x_{T}, y_{T}, z_{T}$ are the Cartesian coordinates of the instantaneous position of the aerial target, and $x, y, z$ are the Cartesian coordinates of the instantaneous position of the missile at time $t_{g}$.

The results of the sample run test are presented in Table 2 and Figures 14-18. The values of missile airframe normal acceleration $a$, angular rate $\omega$, and angle of attack $\alpha$ given in Table 2 are compared for the time instant $t_{g}$.

Table 2. Simulation results for the sample run test.

\begin{tabular}{cccccccc}
\hline $\begin{array}{c}\text { Missile } \\
\text { Number }\end{array}$ & $\begin{array}{c}\text { Gain } \\
\text { Matrix }\end{array}$ & $\begin{array}{c}\boldsymbol{t}_{\boldsymbol{g}} \\
(\mathbf{s})\end{array}$ & $\begin{array}{c}\boldsymbol{d} \\
(\mathbf{m})\end{array}$ & $\begin{array}{c}\boldsymbol{a}\left(\boldsymbol{t}_{\boldsymbol{g}}\right) \\
\left(\mathbf{m} / \mathbf{s}^{2}\right)\end{array}$ & $\begin{array}{c}\omega\left(\boldsymbol{t}_{g}\right) \\
(\mathbf{r a d} / \mathbf{s})\end{array}$ & $\begin{array}{c}\alpha\left(\boldsymbol{t}_{g}\right) \\
(\mathbf{r a d})\end{array}$ & $\begin{array}{c}\boldsymbol{\Theta} \\
(\mathbf{r a d})\end{array}$ \\
\hline 1 & $\mathbf{K}_{1}$ & 1.68 & 3.51 & 163.28 & 0.170 & 0.029 & 0.4009 \\
2 & $\mathbf{K}_{2}$ & 1.68 & 3.51 & 161.05 & 0.136 & 0.029 & 0.3608 \\
3 & $\mathbf{K}_{3}$ & 1.68 & 3.51 & 164.26 & 0.154 & 0.029 & 0.3615 \\
4 & $\mathbf{K}_{4}$ & 1.68 & 4.22 & -102.01 & -0.459 & -0.032 & 0.4604 \\
5 & $\mathbf{K}_{5}$ & 1.68 & 3.51 & 159.16 & 0.090 & 0.029 & 0.3744 \\
6 & $\mathbf{K}_{6}$ & 1.61 & 242.53 & - & - & - & 6.6262 \\
7 & $\mathbf{K}_{7}$ & 1.61 & 242.48 & - & - & - & 6.6212 \\
8 & $\mathbf{K}_{8}$ & 1.68 & 3.51 & 155.68 & 0.067 & 0.029 & 0.3751 \\
\hline
\end{tabular}

The following conclusions can be drawn based on the simulation results.

Although in Section 2.4 values were indicated for the tuning parameter $\zeta$, for which the gain matrices $\mathbf{K}_{6}$ and $\mathbf{K}_{7}$ ought potentially to provide limited stability for the missile airframe, it was shown in the simulation tests that the proposed forms of $\mathbf{K}_{6}$ and $\mathbf{K}_{7}$ do not ensure the required properties of the stabilization system within the entire guidance loop (Figure 14). For this reason, these solutions were excluded from further considerations. 

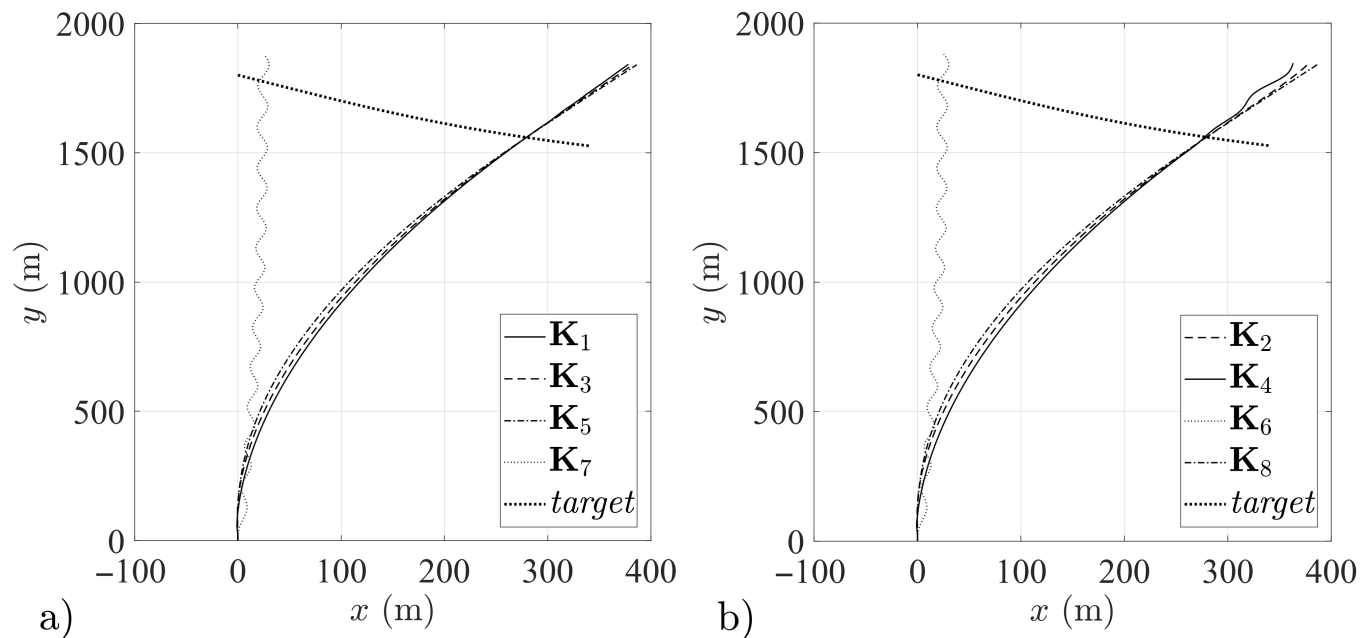

Figure 14. Missile and target trajectories for: (a) missiles 1, 3, 5 and 7, (b) missiles 2, 4, 6 and 8 .
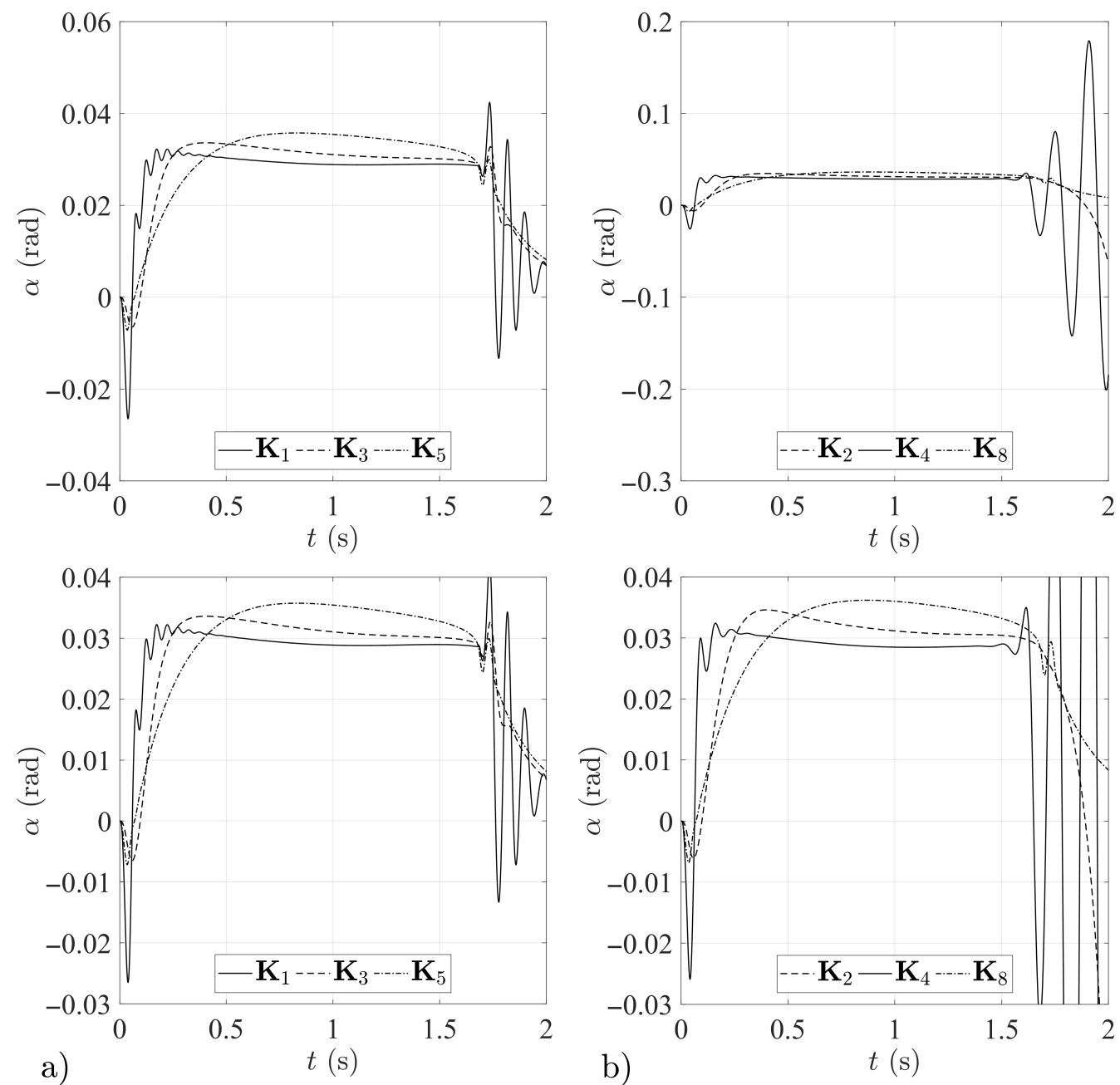

Figure 15. Angle of attack histories for: (a) missiles 1, 3 and 5, (b) missiles 2, 4 and 8. 

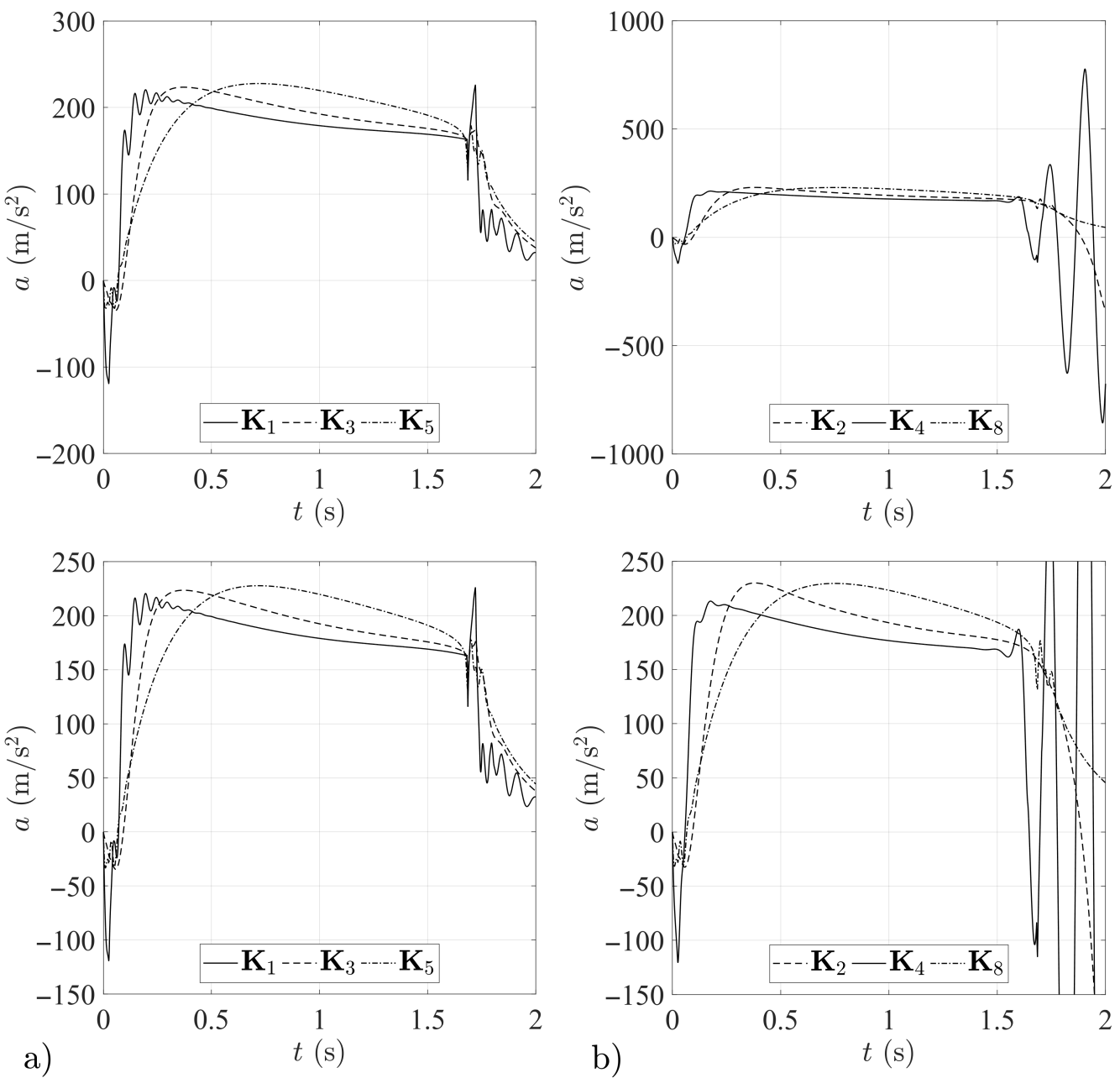

Figure 16. Airframe normal acceleration histories for: (a) missiles 1, 3 and 5, (b) missiles 2, 4 and 8.

In the case of the gain matrices $\mathbf{K}_{1}$ and $\mathbf{K}_{4}$, tests confirmed the oscillations predicted in Section 2.4, which appeared in the initial phase of guidance towards an aerial target. Over the time of the simulation the oscillations are suppressed (Figures 15-18), but the fact of their occurrence is an unfavorable phenomenon and excludes the matrices $\mathbf{K}_{1}$ and $\mathbf{K}_{4}$ from the set of desired solutions. This is an interesting observation, leading to the conclusion that it is not appropriate to design individual elements of the system while neglecting properties of the guidance loop as a whole.

It should be noted, however, that in the case under consideration the presence of these oscillations, in spite of the increase in the value of the index $\Theta$, did not have a significant impact on miss distances (cf. Table 2). Moreover, missiles 1 and 4 attained the smallest instantaneous values of angle of attack (Figure 15) and normal acceleration (Figure 16) among all of the cases considered.

The gain matrices $\mathbf{K}_{2}, \mathbf{K}_{3}, \mathbf{K}_{5}$ and $\mathbf{K}_{8}$ ensure the correct operation of the airframe stabilizing loop. They correctly perform the task of regulation within the entire guidance loop. It may be noted here that the solution pair $\mathbf{K}_{5}-\mathbf{K}_{8}$ produces greater inertia of operation than the pair $\mathbf{K}_{2}-\mathbf{K}_{3}$. This manifested in the higher values of the angular rates (Figure 17), angles of attack (Figure 15) and normal accelerations (Figure 16) attained by missiles 5 and 8. In this way, their regulation systems attempt to make up for the initial losses related to the delayed time of response to the input signal. Finally, however, in the case of all four missiles (numbers 2, 3, 5 and 8) the same values were obtained for the miss distances and guidance times, although the $\Theta$ values indicate the slight superiority of missiles 2 and 3 . 

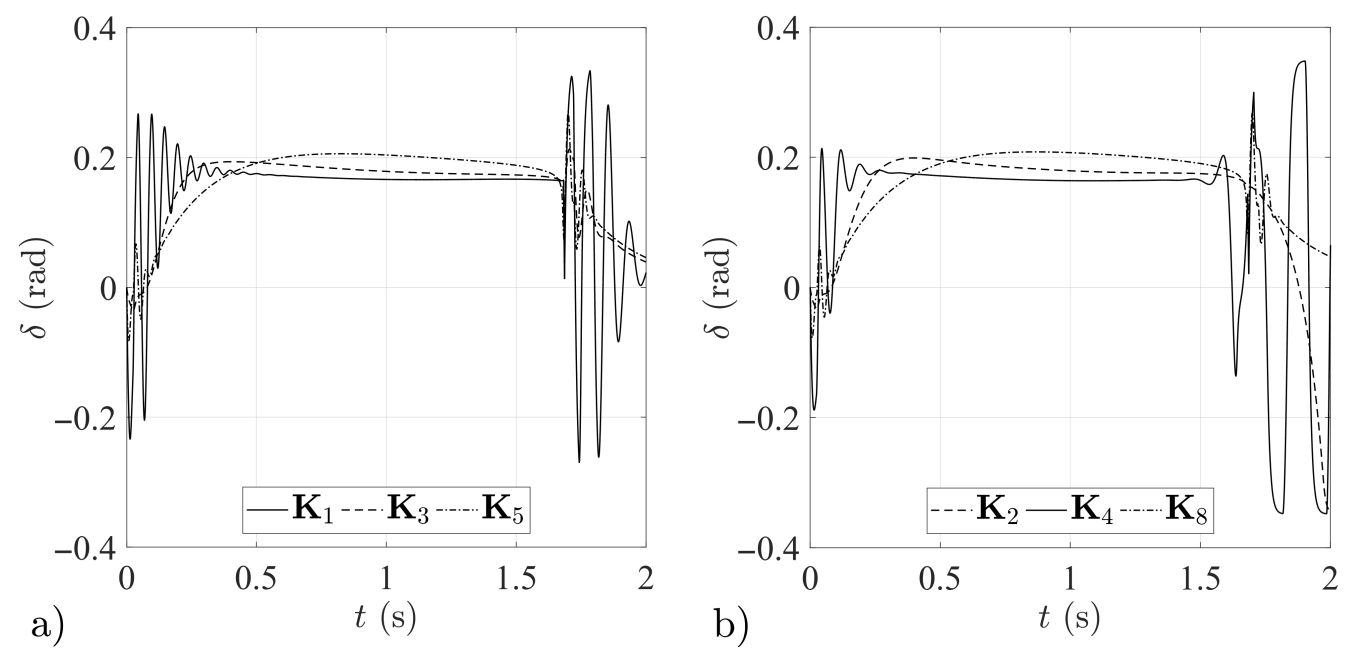

Figure 17. Canard-fin deflection histories for: (a) missiles 1, 3 and 5; (b) missiles 2, 4 and 8.
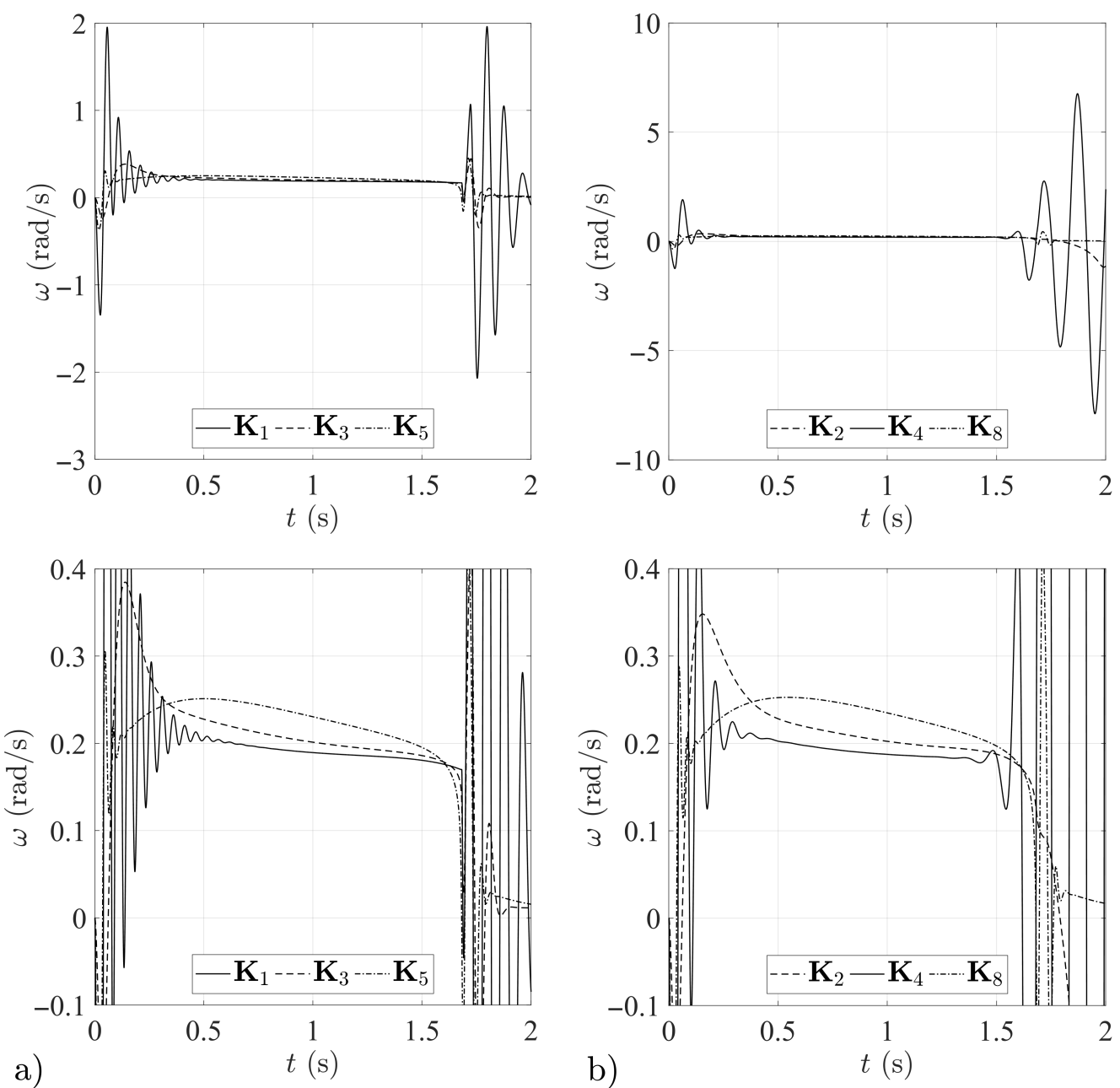

Figure 18. Airframe angular rate histories for: (a) missiles 1,3 and 5; (b) missiles 2, 4 and 8.

\subsection{Monte Carlo Simulation Study}

A Monte Carlo simulation study consisting of 500 sample runs for each missile model was carried out. In these simulations, the aerial target was located at a height of $4000 \mathrm{~m}$ and a distance of $1800 \mathrm{~m}$ from the initial position of the missiles, which was taken as described in Section 3.1. The target control commands performed square-wave evasive maneuvers in 
the pitch and yaw planes with a period of $\Delta T$ and a phase of $\Delta \varphi$ relative to $t=0$. For each test case, the random variables were distributed uniformly and chosen to be: time constant $\tau_{T}=(0.01 \div 0.5) \mathrm{s}$, velocity $V_{T}=(220 \div 340) \mathrm{m} / \mathrm{s}$, initial pitch angle $\theta_{T}=(-\pi / 6 \div \pi / 6)$ rad and initial course angle $\gamma_{T}=(3 \pi / 4 \div 5 \pi / 4)$ rad of the aerial target.

The results of the Monte Carlo simulation study are presented in Figures 19-24. Figures 19a-24a correspond to the results of the simulation in which the proposed stabilization system was treated separately and tested in isolated conditions, where only the phenomena connected with the missile airframe dynamics were taken into account, i.e., for $u=\delta_{\text {com }}=\delta, \dot{\lambda}_{m}=\dot{\lambda}$ and $\Delta \lambda=0$.

Figures $19 \mathrm{~b}-24 \mathrm{~b}$ correspond to the numerical simulation results achieved for the stabilization system tested as a part of the whole guidance loop. The remaining elements of the guidance loop were selected as typical and were not optimized in any way due to the final miss distance or time-to-go. Mean values of guidance results for missiles 1-5 and 8 are given in Tables 3 and 4 . For comparison, the simulation results obtained for the unstabilized airframe (missile 9) are presented, for which only the basic condition of static stability was met (the center of pressure was located behind the center of mass); cf. Figure 25, Tables 3 and 4.
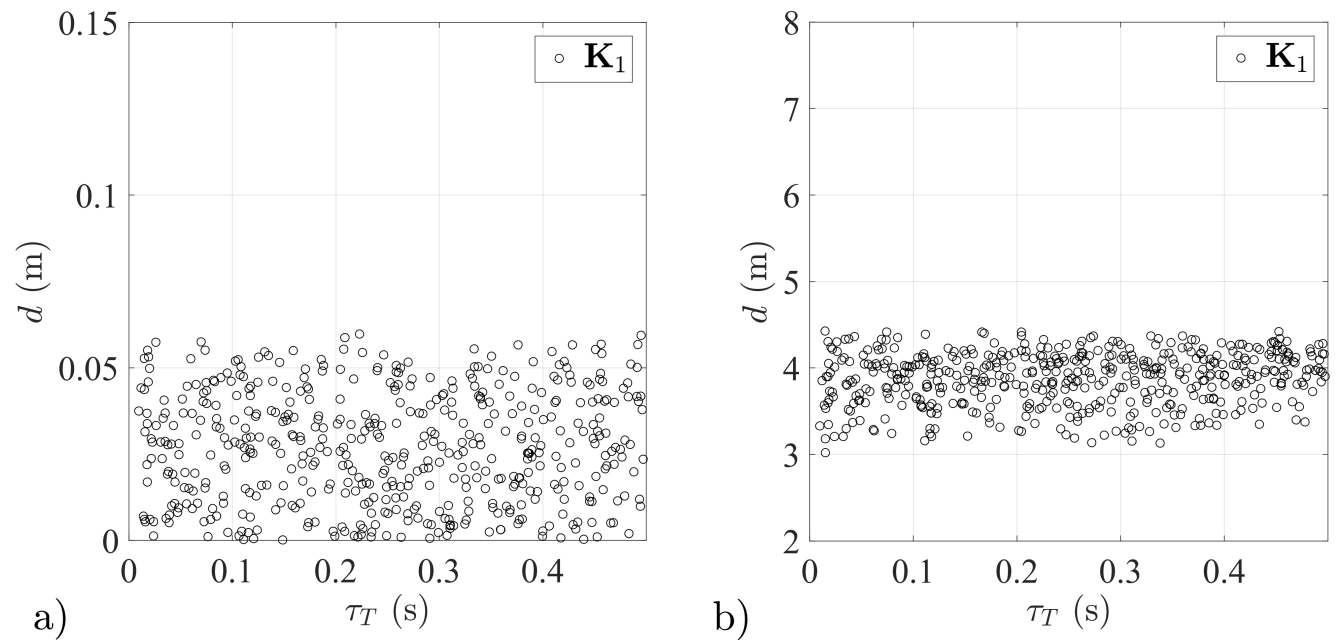

Figure 19. Miss distance distributions for different aerial target dynamics—stabilization system with matrix $\mathbf{K}_{1}$ tested: (a) in isolated conditions, (b) as a part of a guidance loop.
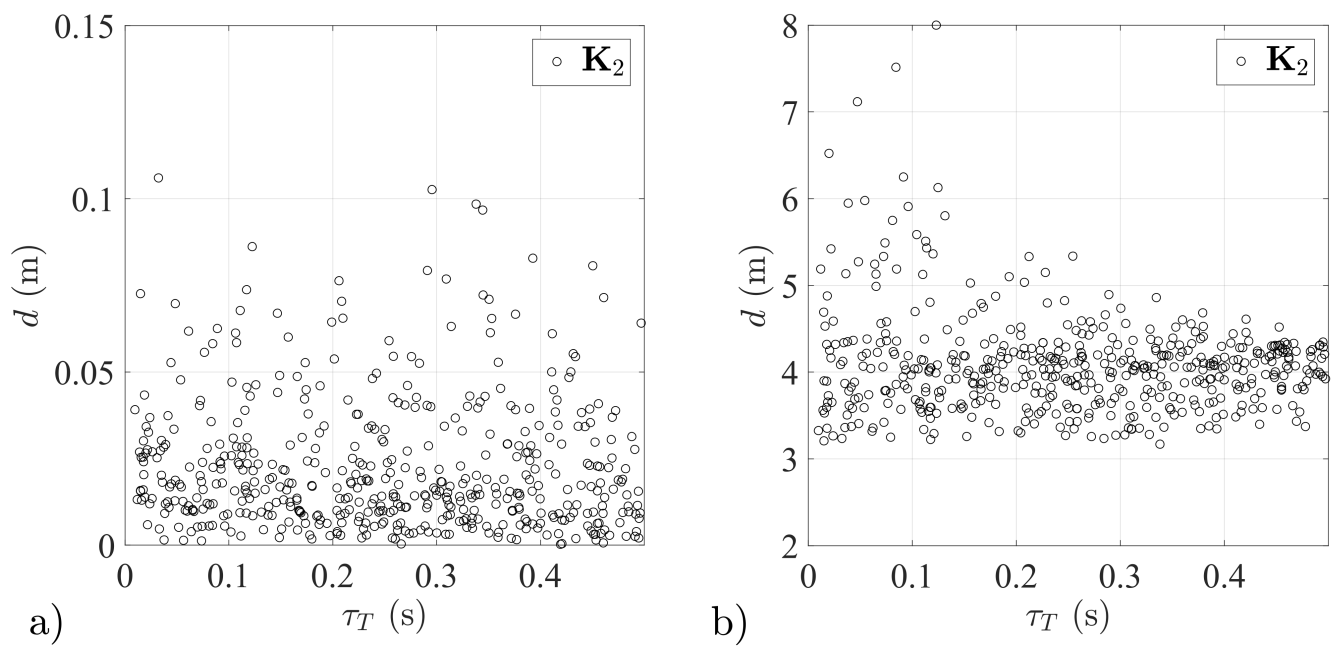

Figure 20. Miss distance distributions for different aerial target dynamics—stabilization system with matrix $\mathbf{K}_{2}$ tested: (a) in isolated conditions, (b) as a part of a guidance loop. 

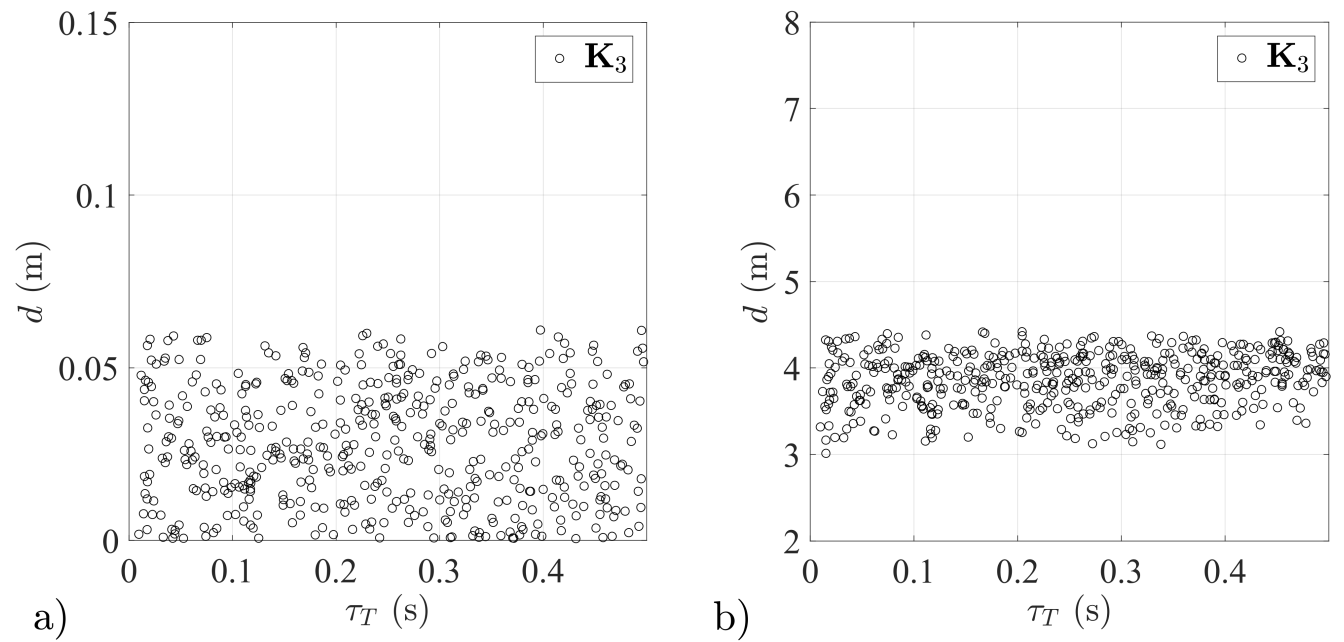

Figure 21. Miss distance distributions for different aerial target dynamics—stabilization system with matrix $\mathbf{K}_{3}$ tested: (a) in isolated conditions, (b) as a part of a guidance loop.
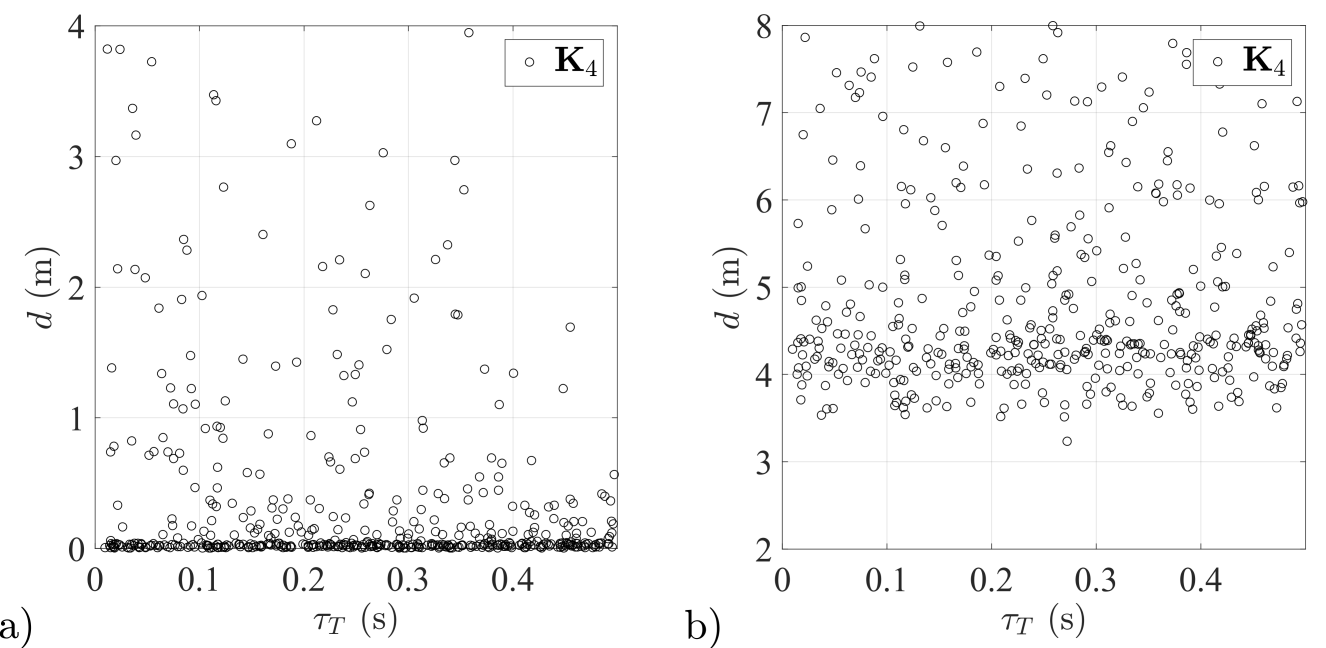

Figure 22. Miss distance distributions for different aerial target dynamics—stabilization system with matrix $\mathbf{K}_{4}$ tested: (a) in isolated conditions, (b) as a part of a guidance loop.
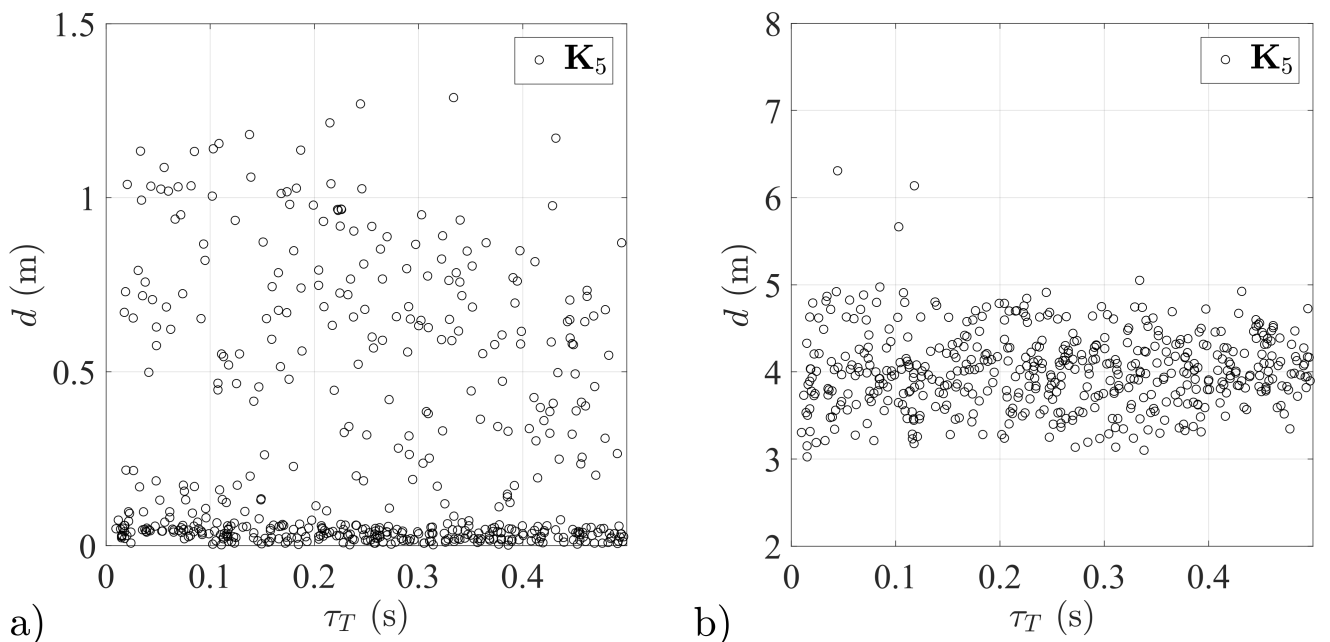

Figure 23. Miss distance distributions for different aerial target dynamics—stabilization system with matrix $\mathbf{K}_{5}$ tested: (a) in isolated conditions, (b) as a part of a guidance loop. 

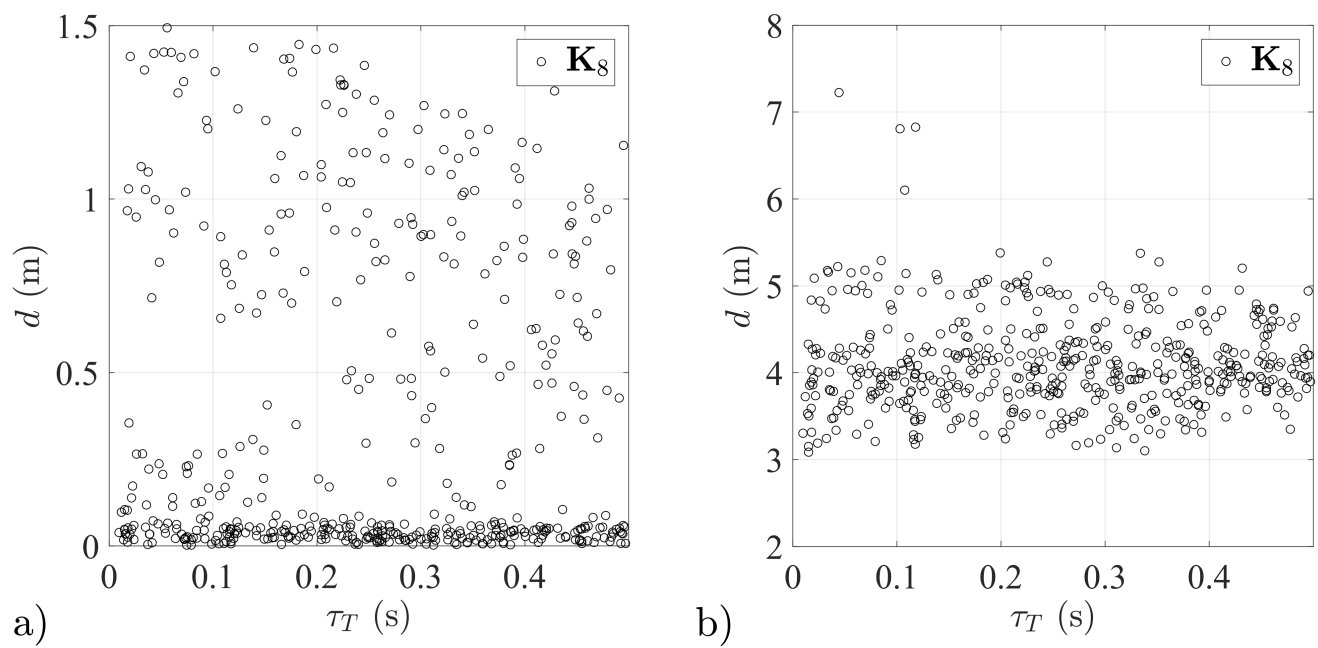

Figure 24. Miss distance distributions for different aerial target dynamics—stabilization system with matrix $\mathbf{K}_{8}$ tested: (a) in isolated conditions, (b) as a part of a guidance loop.
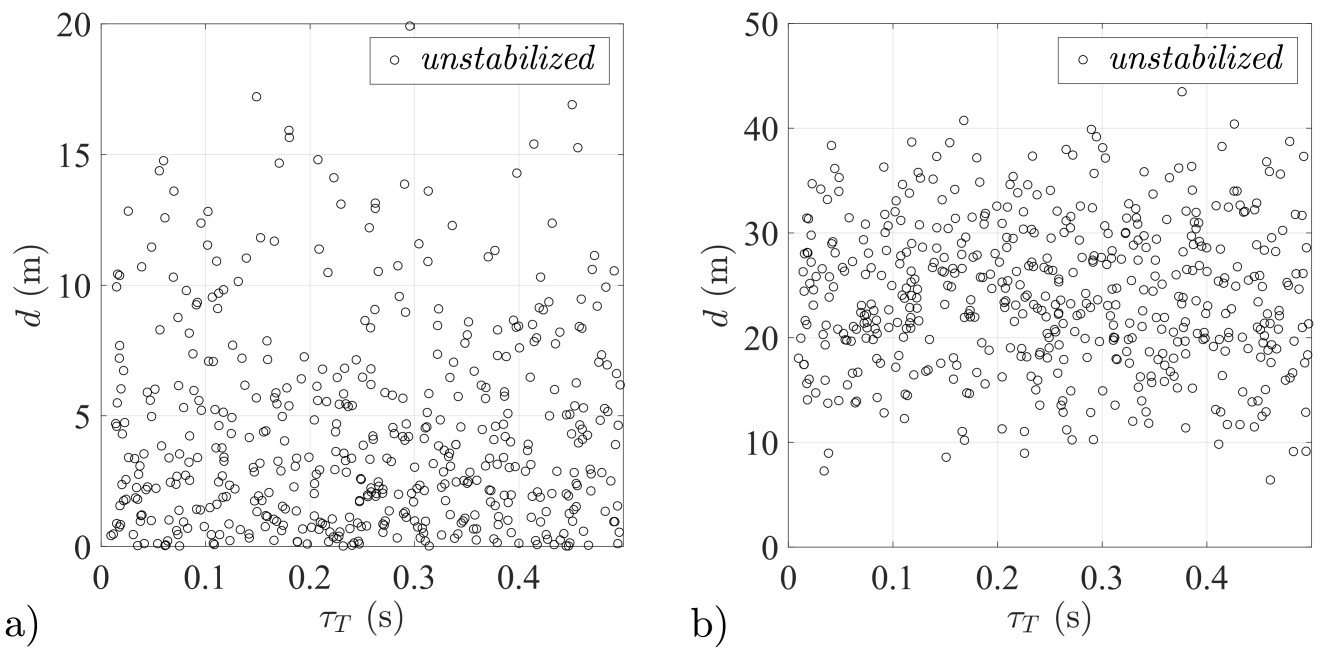

Figure 25. Miss distance distributions for different aerial target dynamics—unstabilized airframe tested: (a) in isolated conditions, (b) as a part of guidance loop.

Table 3. Mean values of guidance results (for isolated conditions).

\begin{tabular}{|c|c|c|c|c|}
\hline Missile Number & Gain Matrix & $\frac{1}{500} \sum_{i=1}^{500} t_{g i}$ & $\frac{1}{500} \sum_{\substack{i=1 \\
(\mathrm{~m})}}^{500} d_{i}$ & $\frac{1}{500} \sum_{i=1}^{500} \Theta_{i}$ \\
\hline 1 & $\mathbf{K}_{1}$ & 1.5536 & 0.0269 & 0.8779 \\
\hline 2 & $\mathbf{K}_{2}$ & 1.5442 & 0.0324 & 0.8034 \\
\hline 3 & $\mathbf{K}_{3}$ & 1.5536 & 0.0259 & 0.8046 \\
\hline 4 & $\mathbf{K}_{4}$ & 1.5535 & 0.6617 & 1.0073 \\
\hline 5 & $\mathbf{K}_{5}$ & 1.5544 & 0.2885 & 0.8369 \\
\hline 8 & $\mathbf{K}_{8}$ & 1.5543 & 0.4090 & 0.8334 \\
\hline 9 & unstabilized & 1.5536 & 5.7028 & 6.3302 \\
\hline
\end{tabular}


Table 4. Mean values of guidance results (for the whole guidance loop).

\begin{tabular}{|c|c|c|c|c|}
\hline Missile Number & Gain Matrix & $\frac{1}{500} \sum_{\substack{i=1 \\
(\mathrm{~s})}}^{500} t_{g i}$ & $\frac{1}{500} \sum_{\substack{i=1 \\
(\mathrm{~m})}}^{500} d_{i}$ & $\frac{1}{500} \sum_{\substack{i=1 \\
(\mathrm{rad})}}^{500} \Theta_{i}$ \\
\hline 1 & $\mathbf{K}_{1}$ & 1.5575 & 3.87 & 0.7433 \\
\hline 2 & $\mathbf{K}_{2}$ & 1.5580 & 4.10 & 0.6692 \\
\hline 3 & $\mathbf{K}_{3}$ & 1.5580 & 3.86 & 0.6705 \\
\hline 4 & $\mathbf{K}_{4}$ & 1.5576 & 5.44 & 0.8537 \\
\hline 5 & $\mathbf{K}_{5}$ & 1.5585 & 4.07 & 0.6946 \\
\hline 8 & $\mathbf{K}_{8}$ & 1.5553 & 4.17 & 0.6945 \\
\hline 9 & unstabilized & 1.5609 & 23.99 & 5.3195 \\
\hline
\end{tabular}

The absolute rotation angle $\Theta$ for a Monte Carlo simulation study was taken as

$$
\Theta=\sum_{n=0}^{N}\left[\frac{1}{f_{s}}\left(\left|\omega_{\theta}(n)\right|+\left|\omega_{\gamma}(n)\right|\right)\right]
$$

where $\omega_{\theta}$ and $\omega_{\gamma}$ are the missile airframe angular rates in the pitch and yaw planes $(\mathrm{rad} / \mathrm{s})$, respectively.

The following conclusions can be drawn based on the simulation results.

The greatest effectiveness of the guidance processes was obtained, in line with expectations, for missile number 3 , with a stabilization system configured based on the gain matrix $\mathbf{K}_{3}$. It should be noted that in the case under consideration, not only was the smallest mean miss distance obtained (cf. Tables 3 and 4 ) but the scatter of miss distances around the mean value was also the smallest. Moreover, this scatter tends to decrease with increasing values of the time constant $\tau_{T}$ characterizing the aerial target dynamics (Figure 21b).

In line with prior expectations, missile number 2, with a stabilization system based on matrix $K_{2}$, exhibited reduced effectiveness in the case of guidance to aerial targets with a short time constant $\tau_{T}$ (for $\tau_{T}>0.4 \mathrm{~s}$ the results are analogous to those for missile number 3; compare Figures 20 and 21). This led to an increase in the mean miss distance.

Surprisingly high effectiveness was achieved in the case of missile number 1 , stabilized by means of a system with matrix $\mathbf{K}_{1}$ (Figure 19). In spite of the imperfections of this solution as outlined in Section 3.1, the system enabled the execution of guidance processes to an aerial target with an effectiveness equal to that of the system using matrix $\mathbf{K}_{3}$ (compare Figures 19 and 21). The following proved to be key factors in the case of missile number 1: rapid reaction of the system to a dynamically changing combat situation, and the ability to suppress undesired oscillations effectively.

The stabilization systems configured based on matrices $\mathbf{K}_{5}$ and $\mathbf{K}_{8}$ had higher mean miss distances than those based on matrices $\mathbf{K}_{3}$ and $\mathbf{K}_{1}$. In the case of missiles 5 and 8 , as for missiles 1, 2 and 3, a reduction was observed in the scatter of the miss distances around the mean value as the time constant $\tau_{T}$ increased (Figures 23 and 24).

The solution using the gain matrix $\mathbf{K}_{4}$ is to be rejected (cf. Figure 22).

It can be seen that for each of tested systems the miss distance was reduced by an order of magnitude with a radical reduction of the airframe rotation. It must be highlighted that the proposed airframe stabilization system is just a part of the autopilot and is not able to reduce the miss distance to zero. Its main task is to protect the on-board seeker before unexpected and rapid dynamic changes, which exert a negative influence on the targettracking process. The non-zero miss distances observed in Figures $19 \mathrm{~b}-24 \mathrm{~b}$ can be explained by the fact that there are inertial components in the assumed guidance loop, e.g., from the seeker drives and actuators. Moreover, the simplest version of the proportional navigation method is used. The missiles are not guided to an overtaken point, so there are always lags behind the indications, which are evident in the case of maneuvering targets. A zero miss 
distance in this situation would only be achieved were the angular rate of the LOS equal to zero.

\section{Conclusions}

This paper has described the use of an adaptive linear-quadratic regulator to stabilize the static and dynamic characteristics of the airframe of a canard-controlled anti-aircraft missile. A procedure to determine analytically the entries of the feedback-loop gain matrix was outlined and a novel method of LQR tuning via single parameter $\varsigma$ was proposed and tested. The main effort was directed towards describing a procedure for selecting appropriate values of parameter $\varsigma$ for the designed airframe stabilization system, and presenting the results of the simulation tests. It was observed moreover, that the solutions obtained are symmetric pairs, and that the tuning parameter $\varsigma$ proposed for the designed adaptive linear-quadratic regulator enables the selection of suitable parameters for the airframe stabilizing loop for the majority of the analytical solutions of the considered matrix, the Riccati equation.

The results confirm that the analytical methods initially applied in $[7,8]$ are correct. Likewise, they confirm the implication that the solutions found to the equations should display symmetry. In the case of six of the eight analytical solutions, it was possible to find values of the tuning parameter $\varsigma$ for which the system with a linear-quadratic regulator effectively performs the task of stabilizing the missile airframe. It should be noted, however, that not all of these solutions also provide effective guidance of the missile to an aerial target. Rather, this is a result of the properties of the guidance loop as a whole.

As mentioned at the beginning of the paper, in the aerospace field, for the applications of the $\mathrm{LQR}$, the $\mathbf{Q}$ and $\mathbf{R}$ matrices are usually determined based on Bryson principle (or modified, e.g., time-varying Bryson) or "trial and error" methods. The main problem connecting all of these is the need to define the $Q$ values to find the solution of the feedback gain matrix $\mathbf{K}$. In the proposed concept, a basic formula of feedback loop gain matrix equations (without defining parameter $\zeta$, or-more precisely from the point of view of this paper-for tuning parameter $\zeta=1$ in covert form) for the time-varying linear-quadratic stabilization system eliminates the need to choose $\mathbf{Q}$ values at all, as was shown in [7]. However, the approach proposed in [7] does not use all the available pairs of equations (i.e., potential solutions) describing the required operation of the stabilization system. In previous works [7,8], these pairs of equations, which give unstable solutions (with positive feedback) or which lead to inappropriate system response quality, were rejected. Here, by introducing a single tuning parameter $\varsigma$ into the equations describing the entries of gain matrix $\mathbf{K}$, all of the analytical solutions can be examined and fully exploited against assumed requirements.

Obtaining an analytical solution for an $L Q R$ with a single tuning parameter is very attractive from a practical (applicational) point of view. First of all, the basic form of an adaptive controller is obtained naturally. Secondly, the tuning procedure is reduced to the selection of one parameter instead of the whole matrix. The disadvantage of the proposed method is that the condition for its application is to find an analytical solution of the controller, which in general cases is difficult or impossible, e.g., because of obtaining systems of equations in entangled forms. For this reason, the discussed concept may constitute a solution for some specific control systems (those for which it is possible to find a solution in analytical form), but it cannot be treated as a universal method of designing LQR controllers.

The proposed method could be useful, e.g., when upgrading anti-aircraft missile systems of previous generations, enabling a significant increase in the capabilities of the missile with relatively negligible hardware and software interference. The algorithm is relatively easy to implement in practice. Since the coefficients given by Equation (8) are generally functions of missile velocity, with knowledge of the velocity profile, the entries of gain matrix $\mathbf{K}$ can be found for successive time instants via preprocessing mode and then tabulated or approximated by polynomial functions. Moreover, knowledge of the 
airframe angular rate $\omega$ and the angle of attack $\alpha$ is required. The actual value of $\omega$ in the control plane is supplied by an angular rate gyroscope. The value of $\alpha$ may be determined directly or indirectly: by using an on-board instrument for measuring the angle of attack or by means of Equation (10) where the actual angle of the airframe in the control plane is supplied by a free gyroscope, whereas the angle of the velocity vector is obtained by integrating, with respect to time, the ratio of the airframe acceleration in the control plane to the missile velocity module. Both approaches give rise to certain technical and implementation problems. Discussion of these issues is beyond the scope of this paper.

However, it is necessary to note the potential dangers of the proposed approach, especially from the robustness point of view. After linearization, the control action can be very sensitive to omitted high-order terms; i.e., linear systems are not stable in a Lyapunov sense. In this paper, by calculating the feedback loop gain matrix $\mathbf{K}$ based on the time-varying parameters and tuning parameter $\varsigma$, an adaptive-tuned linear-quadratic stabilization system design is proposed to deal with the various disturbances affecting the operation of the seeker during the missile-target engagement process. The fact that the missile airframe behaves as a natural low-pass filter gives some additional protection (the airframe bandwidth, depending on the missile class, is usually in the range from a few to several $\mathrm{Hz}$ ). This airframe dynamics feature quite effectively removes unwanted distortions and reduces the impacts of high order terms on the guidance process. Nevertheless, under certain unfavorable conditions, the stability of the whole system could be potentially violated. Therefore, further work should take into account the development of the proposed stabilization system towards adaptive linear robust control under external disturbance effects. From this point of view, the analysis based on the attractive ellipsoid method (AEM) seems adequate to the problem under consideration, as a methodology providing robust stabilization of dynamic systems [50,51]. In this approach, use of the dynamic model of the system is not necessary. Instead, the AEM uses basic information (i.e., the number of state variables, the number of measurable outputs and the bounds of disturbances), while providing practical stability and guaranteeing that each of the possible trajectories of the closed-loop system achieves an ellipsoid of a minimal size [50]. Future work will also be aimed at searching for a solution for the system of equations, taking into account the inertia caused by fin servos and attempting to generalize the proposed solution for the case of the pitch-yaw-roll-integrated stabilization system.

Funding: I have not received external funding.

Institutional Review Board Statement: Not applicable.

Informed Consent Statement: Not applicable.

Data Availability Statement: Sample data available on request.

Conflicts of Interest: I declare no conflict of interest.

\section{Appendix A}

The closed-loop system described by Equation (13) is given as:

$$
\dot{\mathbf{x}}=(\mathbf{A}-\mathbf{B K}) \mathbf{x}+\mathbf{B} u
$$

with output equation

$$
\mathbf{y}=\mathbf{C} \mathbf{x}+\mathbf{D} u \rightarrow\left[\begin{array}{l}
y_{\alpha} \\
y_{\omega}
\end{array}\right]=\left[\begin{array}{l}
\mathbf{C}_{\alpha} \\
\mathbf{C}_{\omega}
\end{array}\right]\left[\begin{array}{l}
\alpha \\
\omega
\end{array}\right]+\left[\begin{array}{l}
0 \\
0
\end{array}\right] u \text { where } \quad \mathbf{C}=\left[\begin{array}{l}
\mathbf{C}_{\alpha} \\
\mathbf{C}_{\omega}
\end{array}\right]=\left[\begin{array}{ll}
1 & 0 \\
0 & 1
\end{array}\right]
$$

The transfer function due to the $\omega$ takes the form

$$
\frac{Y_{\omega}(s)}{U(s)}=\mathbf{C}_{\omega}[s \mathbf{I}-(\mathbf{A}-\mathbf{B K})]^{-1} \mathbf{B}
$$


The full-state feedback system does not compare the output to the reference. Instead, it compares the state vector $\mathbf{x}$ multiplied by the gain matrix $\mathbf{K}$ to the reference. To obtain the desired output, the reference input must be scaled:

$$
u_{r e f}=N u-\mathbf{K} \mathbf{x}
$$

where $N$ is the extra gain used to scale the closed-loop transfer function. Now we have:

$$
\begin{gathered}
\dot{\mathbf{x}}=(\mathbf{A}-\mathbf{B K}) \mathbf{x}+\mathbf{B} N u \\
\frac{Y_{\omega}(s)}{U_{r e f}(s)}=\mathbf{C}_{\omega}[s \mathbf{I}-(\mathbf{A}-\mathbf{B K})]^{-1} \mathbf{B} N=G_{\text {closed }}(s) N
\end{gathered}
$$

It can be computed that

$$
\begin{gathered}
N=G_{\text {closed }}(0)^{-1}=-\left[\mathbf{C}_{\omega}(\mathbf{A}-\mathbf{B K})^{-1} \mathbf{B}\right]^{-1} \\
N=-\left\{\left[\begin{array}{ll}
0 & 1
\end{array}\right]\left(\left[\begin{array}{cc}
-a_{1} & 1 \\
-a_{2} & -a_{3}
\end{array}\right]-\left[\begin{array}{l}
0 \\
b
\end{array}\right]\left[\begin{array}{ll}
k_{1} & k_{2}
\end{array}\right]\right)^{-1}\left[\begin{array}{l}
0 \\
b
\end{array}\right]\right\}^{-1} \\
N=-\left\{\left[\begin{array}{ll}
0 & 1
\end{array}\right]\left[\begin{array}{c}
\frac{-b}{a_{2}+a_{1} a_{3}+b k_{1}+a_{1} b k_{2}} \\
\frac{-a_{1} b}{a_{2}+a_{1} a_{3}+b k_{1}+a_{1} b k_{2}}
\end{array}\right]\right\}^{-1} \\
N=\underbrace{\frac{a_{2}+a_{1} a_{3}+b k_{1}+a_{1} b k_{2}}{a_{1} b}}_{N_{u}}+\underbrace{\left[\begin{array}{ll}
k_{1} & k_{2}
\end{array}\right]}_{\mathbf{K}} \underbrace{\left[\begin{array}{c}
\frac{1}{a_{1}} \\
1
\end{array}\right]}_{\mathbf{N}_{x}}=N_{u}+\mathbf{K N}_{x}
\end{gathered}
$$

By substituting (A11) into (A4), Equation (31) is obtained. By closing $N_{u}$ and $\mathbf{N}_{x}$ in an elegant matrix form $[28,52]$, we get

$$
\begin{aligned}
& {\left[\begin{array}{l}
\mathbf{N}_{x} \\
N_{u}
\end{array}\right]=\mathbf{\Omega}^{-1}\left[\begin{array}{l}
0 \\
1
\end{array}\right] \quad \text { where } \quad \mathbf{\Omega}=\left[\begin{array}{cc}
\mathbf{A} & \mathbf{B} \\
\mathbf{C}_{\omega} & 0
\end{array}\right]=\left[\begin{array}{ccc}
-a_{1} & 1 & 0 \\
-a_{2} & -a_{3} & b \\
0 & 1 & 0
\end{array}\right]} \\
& {\left[\begin{array}{l}
\mathbf{N}_{x} \\
N_{u}
\end{array}\right]=\left[\begin{array}{ccc}
\frac{-1}{a_{1}} & 0 & \frac{1}{a_{1}} \\
0 & 0 & 1 \\
\frac{-a_{2}}{a_{1} b} & \frac{1}{b} & \frac{a_{2}+a_{1} a_{3}}{a_{1} b}
\end{array}\right]\left[\begin{array}{l}
0 \\
0 \\
1
\end{array}\right]=\left[\begin{array}{c}
\frac{1}{a_{1}} \\
1 \\
\frac{a_{2}+a_{1} a_{3}}{a_{1} b}
\end{array}\right]} \\
& {\left[\begin{array}{c}
\mathbf{N}_{x} \\
N_{u}
\end{array}\right]=\left[\begin{array}{c}
{\left[\begin{array}{c}
1 \\
a_{1} \\
1
\end{array}\right]} \\
\frac{a_{2}+a_{1} a_{3}}{a_{1} b}
\end{array}\right]}
\end{aligned}
$$

which is consistent with Equations (A11) and (32).

\section{Appendix B}

For simulation purposes, the aerial target is modeled as a mass point $[6,41,45,53-55]$ whose motion in space is described by the vector of parameters

$$
\mathbf{T} \in \mathbb{R}^{1 \times 7}: \quad \mathbf{T}=\left[\begin{array}{lllllll}
x_{T} & y_{T} & z_{T} & \theta_{T} & \gamma_{T} & \phi_{T} & V_{T}
\end{array}\right]
$$


where $x_{T}, y_{T}, z_{T}$ are the coordinates of the target's position $(\mathrm{m}) ; \theta_{T}$ and $\gamma_{T}$ are the pitch and yaw angles of the target's velocity vector (rad), respectively; $\phi_{T}$ is the roll angle (rad); $V_{T}$ is the modulus of the target's velocity vector $(\mathrm{m} / \mathrm{s})$. Changes in the target's accelerations are approximated by the first-order dynamics

$$
\dot{a}_{L}=\frac{1}{\tau_{T}}\left(u_{L}-a_{L}\right) \quad \dot{a}_{\theta}=\frac{1}{\tau_{T}}\left(u_{\theta}-a_{\theta}\right) \quad \dot{a}_{\gamma}=\frac{1}{\tau_{T}}\left(u_{\gamma}-a_{\gamma}\right)
$$

where $u_{L}$ is the commanded longitudinal acceleration $\left(\mathrm{m} / \mathrm{s}^{2}\right) ; u_{\theta}$ and $u_{\gamma}$ are the manoeuvre commands in the pitch and yaw plane $\left(\mathrm{m} / \mathrm{s}^{2}\right)$, respectively; $a_{L}$ is the actual longitudinal acceleration $\left(\mathrm{m} / \mathrm{s}^{2}\right) ; a_{\theta}$ and $a_{\gamma}$ are the actual accelerations in the pitch and yaw plane $\left(\mathrm{m} / \mathrm{s}^{2}\right)$, respectively; $\tau_{T}$ is the time constant of the target's dynamics $(\mathrm{s})$. The manoeuvre commands are subject to limitations in the form

$$
\left|u_{L}\right| \leq \bar{u}_{L} \quad\left|u_{\theta}\right| \leq \bar{u}_{\theta} \quad\left|u_{\gamma}\right| \leq \bar{u}_{\gamma}
$$

Changes in the target's velocity and in the pitch and yaw angles are defined as

$$
\dot{V}_{T}=a_{L} \quad \dot{\theta}_{T}=\frac{a_{\theta}}{V_{T}} \quad \dot{\gamma}_{T}=\frac{a_{\gamma}}{V_{T}}
$$

while the roll angle is approximated by

$$
\phi_{T}=\arctan \left(\frac{V_{T}}{g} \dot{\gamma}_{T}\right)=\arctan \left(\frac{a_{\gamma}}{g}\right)
$$

where $g$ is the gravitational acceleration $\left(\mathrm{m} / \mathrm{s}^{2}\right)$. The following kinematic equations for the motion of the target's center of mass are used:

$$
\dot{x}_{T}=V_{T} \cos \theta_{T} \sin \gamma_{T} \quad \dot{y}_{T}=V_{T} \sin \theta_{T} \quad \dot{z}_{T}=V_{T} \cos \theta_{T} \cos \gamma_{T}
$$

\section{References}

1. Holloway, J.C.; Krstic, M. A Predictor Observer for Seeker Delay in the Missile Homing Loop. IFAC Pap. 2015, 48, 416-421. [CrossRef]

2. Kaiwei, C.; Qunli, X.; Xiao, D.; Yuemin, Y. Influence of Seeker Disturbance Rejection and Radome Error on the Lyapunov Stability of Guidance Systems. Math. Probl. Eng. 2018, 2018, 1890426. [CrossRef]

3. Lin, S.; Lin, D.; Wang, W. A Novel Online Estimation and Compensation Method for Strapdown Phased Array Seeker Disturbance Rejection Effect Using Extended State Kalman Filter. IEEE Access 2019, 7, 172330-172340. [CrossRef]

4. Yunli, D.; Qunli, X.; Zhuomin, W. Effect of seeker disturbance rejection performance on the control system stability. In Proceedings of the 3rd International Symposium on Systems and Control in Aeronautics and Astronautics, Harbin, China, 8-10 June 2010. [CrossRef]

5. Shixiang, L.; Tianyu, L.; Teng, S.; Qunli, X. Dynamic Modeling and Coupling Characteristic Analysis of Two-Axis Rate Gyro Seeker. Int. J. Aerosp. Eng. 2018, 2018, 8513684. [CrossRef]

6. Grycewicz, H.; Mosiewicz, R.; Pietrasieński, J. Radio Control Systems, 1st ed.; Military University of Technology: Warsaw, Poland, 1984. (In Polish)

7. Bużantowicz, W. A sliding mode controller design for a missile autopilot system. J. Theor. Appl. Mech. 2020, 58, 169-182. [CrossRef]

8. Bużantowicz, W. A linear-quadratic stabilization system for a canard-controlled missile. In Proceedings of the 25th International Conference “Engineering Mechanics 2019”, Svratka, Czech Republic, 13-16 May 2019; pp. 73-76. [CrossRef]

9. Åström, K.J.; Kumar, P.R. Control: A perspective. Automatica 2014, 50, 3-43. [CrossRef]

10. Ogata, K. Modern Control Engineering, 5th ed.; Prentice Hall: New York, NY, USA, 2010.

11. Alameri, S.; Lazic, D.; Ristanovic, M. A Comparative Study of PID, PID with Tracking, and FPID Controller for Missile Canard with an Optimized Genetic Tuning Method Using Simscape Modelling. Teh. Vjestn. 2018, 25 (Suppl. 2), 427-436. [CrossRef]

12. Kada, B. A New Methodology to Design Sliding-PID Controllers: Application to Missile Flight Control System. IFAC Proc. Vol. 2012, 45, 673-678. [CrossRef]

13. Kianfar, K.; Amiri, R.; Bozorgmehr, A. Designing the Non-Linear PID Controller for a Missile. In Proceedings of the 1st International Conference on Informatics and Computational Intelligence, Bandung, Indonesia, 12-14 December 2011; pp. 171-175. [CrossRef] 
14. Zhang, W.; Lei, J.; Shi, X.; Liang, G. Research on Robustness of Double PID Control of Supersonic Missiles. In Information Computing and Applications. ICICA 2011. Communications in Computer and Information Science; Liu, C., Chang, J., Yang, A., Eds.; Springer: Berlin/Heidelberg, Germany, 2011; Volume 244. [CrossRef]

15. Aboelela, M.A.S.; Ahmed, M.F.; Dorrah, H.T. Design of aerospace control systems using fractional PID controller. J. Adv. Res. 2012, 3, 225-232. [CrossRef]

16. Ahmed, M.F.; Dorrah, H.T. Design of gain schedule fractional PID control for nonlinear thrust vector control missile with uncertainty. Automatika 2018, 59, 357-372. [CrossRef]

17. Pan, B.; Fareed, U.; Qing, W.; Tian, S. A novel fractional order PID navigation guidance law by finite time stability approach. ISA Trans. 2019, 94, 80-92. [CrossRef]

18. Çimen, T. A Generic Approach to Missile Autopilot Design using State-Dependent Nonlinear Control. IFAC Proc. Vol. 2011, 44, 9587-9600. [CrossRef]

19. Menon, P.K.; Ohlmeyer, E.J. Integrated design of agile missile guidance and autopilot systems. Control Eng. Pract. 2001, 9, 1095-1106. [CrossRef]

20. Chen, J.; Xiong, Z.; Liang, Z.; Bai, C.; Yi, K.; Ren, Z. Hypersonic Vehicles Profile-Following Based on LQR Design Using TimeVarying Weighting Matrices. In Advances in Some Hypersonic Vehicles Technologies, 1st ed.; Agarwal, R.K., Ed.; IntechOpen: London, UK, 2018; pp. 133-151. [CrossRef]

21. Nocon, Ł.; Koruba, Z. Modified linear-quadratic regulator used for controlling anti-tank guided missile in vertical plane. J. Theor Appl. Mech. 2020, 58, 723-732. [CrossRef]

22. Park, J.; Kim, Y.; Kim, J.H. Integrated Guidance and Control Using Model Predictive Control with Flight Path Angle Prediction against Pull-Up Maneuvering Target. Sensors 2020, 20, 3143. [CrossRef] [PubMed]

23. Talole, S.E.; Godbole, A.A.; Kolhe, J.P.; Phadke, S.B. Robust Roll Autopilot Design for Tactical Missiles. J. Guid. Control Dyn. 2011, 34, 107-118. [CrossRef]

24. Vincent, R.V.; Economou, J.; Wall, D.G.; Cleminson, J. $\mathrm{H}_{\infty} /$ LQR Optimal Control for a Supersonic Air-Breathing Missile of Asymmetric Configuration. IFAC Pap. 2019, 52, 214-218. [CrossRef]

25. Devan, V.; Chandar T.S. Cascaded LQR Control for Missile Roll Autopilot. In Proceedings of the 2018 International CET Conference on Control, Communication, and Computing (IC4), Trivandrum, India, 5-7 July 2018; pp. 45-50. [CrossRef]

26. Wise, K.A. A Trade Study on Missile Autopilot Design Using Optimal Control Theory. In Proceedings of the AIAA Guidance, Navigation and Control Conference and Exhibit, Hilton Head, SD, USA, 20-23 August 2007. [CrossRef]

27. Çimen, T. State-Dependent Riccati Equation (SDRE) Control: A Survey. IFAC Proc. Vol. 2008, 41, 3761-3775. [CrossRef]

28. Franklin, G.F.; Powell, D.J.; Emami-Naeini, A. Feedback Control of Dynamic Systems, 6th ed.; Pearson Education: London, UK, 2011.

29. Åström, K.J.; Murray, R.M. Feedback Systems: An Introduction for Scientists and Engineers, 1st ed.; Princeton University Press: Princeton, NJ, USA, 2012.

30. Deng, X.; Sun, X.; Liu, R.; Wei, W. Optimal analysis of the weighted matrices in LQR based on the differential evolution algorithm. In Proceedings of the 29th Chinese Control and Decision Conference (CCDC), Chongqing, China, 28-30 May 2017 ; pp. 832-836. [CrossRef]

31. Dong-Qing, F. Simulation Research on the Weighting Matrix of the Optimal Linear System. J. Zhengzhou Univ. Technol. 2000, 21, 11-14.

32. Li, Y.; Liu, J.; Wang, Y. Design Approach of Weighting Matrices for LQR Based on Multi-objective Evolution Algorithm. In Proceedings of the 2008 IEEE International Conference on Information and Automation, Changsha, China, 20-23 June 2008; pp. 1188-1192. [CrossRef]

33. Peng, Y.; Chen, Y.Q. Optimal design of the LQR controller based on the improved shuffled frog-leaping algorithm. CAAI Trans. Intell. Syst. 2014, 9, 480-484. [CrossRef]

34. Siddiq, M.K.; Cheng, F.J.; Bo, Y.W. SDRE Based Integrated Roll, Yaw and Pitch Controller Design for 122 mm Artillery Rocket. Appl. Mech. Mater. 2013, 415, 200-208. [CrossRef]

35. Abdelhamid, A.M.; El-Gaafary, A. Optimal Decentralized LQR Control to Enhance Multi-Area LFC System Stability. Math. Probl. Eng. 2018, 2018, 7232915. [CrossRef]

36. Stefanescu, R.M.; Prioroc, C.L.; Stoica, A.M. Weighting matrices determination using pole placement for tracking maneuvers. UPB Sci. Bull. Ser. D Mech. Eng. 2013, 75, 31-40.

37. Siouris, G.M. Missile Guidance and Control Systems, 1st ed.; Springer: New York, NY, USA, 2004.

38. Zarchan, P. Tactical and Strategic Missile Guidance, 6th ed.; AIAA: Reston, VA, USA, 2012.

39. Zaikang, Q.; Defu, L. Design of Guidance and Control Systems for Tactical Missiles, 1st ed.; CRC Press: Boca Raton, FL, USA, 2020.

40. Brugarolas, P.B.; Fromion, V.; Safonov, M.G. Robust switching missile autopilot. In Proceedings of the American Control Conference, Philadelphia, PA, USA, 24-26 June 1998; Volume 6, pp. 3665-3669. [CrossRef]

41. Idan, M.; Shima, T.; Golan, O.M. Integrated Sliding Mode Autopilot-Guidance for Dual Control Missiles. J. Guid. Control Dyn. 2007, 30, 1081-1089. [CrossRef]

42. Lee, H.C.; Choi, J.W.; Song, T.L.; Song, C.H. Agile Missile Autopilot Design via Time-Varying Eigenvalue Assigment. In Proceedings of the 8th International Conference on Control, Automation, Robotics and Vision, Kunming, China, 6-9 December 2004; pp. 1832-1837. [CrossRef] 
43. McLain, T.W.; Beard, R.W. Nonlinear Robust Missile Autopilot Design Using Successive Galerkin Approximation. In Proceedings of the AIAA Guidance, Navigation, and Control Conference and Exhibit, Portland, OR, USA, 9-11 August 1999; pp. 384-391. [CrossRef]

44. Shamma, J.S.; Cloutier, J.R. Gain-Sheduled Missile Autopilot Design Using Linear Parameter Varying Transformations. J. Guid. Control Dyn. 1993, 16, 256-263. [CrossRef]

45. Shima, T.; Idan, M.; Golan, O.M. Sliding-Mode Control for Integrated Missile Autopilot Guidance. J. Guid. Control Dyn. 2006, 29, 250-260. [CrossRef]

46. Zhao, Z.; Jun, H. A High Gain Integral Dynamic Compensation Method for Nonlinear Missile Control. IFAC Proc. Vol. 2011, 44, 2048-2053. [CrossRef]

47. Cronvich, L.L. Missile Aerodynamics. Johns Hopkins APL Tech. Dig. 1983, 4, 175-186.

48. Kurov, V.D.; Dolzhansky, J.M. Principles Behind the Solid-Propellant Rocket Design, 1st ed.; Publishing House of Ministry of National Defense: Warsaw, Poland, 1964. (In Polish)

49. Moore, F. Approximate Methods for Weapon Aerodynamics, 1st ed.; Progress in Astronautics and Aeronautics; AIAA: Reston, VT, USA, 2000; Volume 186. [CrossRef]

50. Ordaz, P.; Poznyak, A. The Furuta's Pendulum Stabilization without the Use of a Mathematical Model: Attractive Ellipsoid Method with KL-adaptation. In Proceedings of the 51st IEEE Conference on Decision and Control (CDC), Maui, HI, USA, 10-13 December 2012; pp. 7285-7290. [CrossRef]

51. Ordaz, P.; Poznyak, A. Adaptive-Robust Stabilization of the Furuta's Pendulum via Attractive Ellipsoid Method. J. Dyn. Syst. Meas. Control 2016, 138, 021005. [CrossRef]

52. Franklin, G.F.; Powell, D.J;; Workman, M.L. Digital Control of Dynamic Systems, 3rd ed.; Addison-Wesley: Menlo Park, CA, USA, 1997.

53. Lin, C.L.; Chen, Y.Y. Design of Fuzzy Logic Guidance Law Against High-Speed Target. J. Guid. Control Dyn. 2000, $23,17-25$. [CrossRef]

54. Bużantowicz, W. Dual-control missile guidance: A simulation study. J. Theor. Appl. Mech. 2018, 56, 727-739. [CrossRef]

55. Bużantowicz, W. A Method of Visualizing Velocity and Acceleration Profiles of an Air Target. Probl. Mechatronics. Armament Aviat. Saf. Eng. 2018, 9, 9-20. [CrossRef] 